\title{
Rutin and ascorbic acid cooperation in antioxidant and antiapoptotic effect on human skin keratinocytes and fibroblasts exposed to UVA and UVB radiation
}

\author{
Agnieszka Gęgotek ${ }^{1} \cdot$ Ewa Ambrożewicz $^{1} \cdot$ Anna Jastrząb $^{1} \cdot$ Iwona Jarocka-Karpowicz $^{1} \cdot$ Elżbieta Skrzydlewska $^{1}$
}

Received: 26 September 2018 / Revised: 12 February 2019 / Accepted: 14 February 2019 / Published online: 19 February 2019

(c) The Author(s) 2019

\begin{abstract}
The combination of ascorbic acid and rutin is frequently used in oral preparations. However, despite numerous protective effects of each component individually, their combined effect on ultraviolet (UV)-irradiated skin cells has never been evaluated. The aim of this study was to evaluate the combined effect of ascorbic acid and rutin on human keratinocytes and fibroblasts exposed to UVA and UVB radiation. Skin keratinocytes and fibroblasts exposed to UVA and UVB radiation were treated with ascorbic acid or/and rutin. The total antioxidant properties of both components, as well as their effect on cellular pro- and antioxidant status, lipid and protein oxidation, transmembrane transport, and pro-inflammatory and pro/ antiapoptotic protein expression were measured. The combination of ascorbic acid and rutin had higher antioxidant properties compared to the activity of the single compound alone, and showed a stronger effect against UV-induced reactive oxygen species generation. The ascorbic acid and rutin combination also showed increased antioxidant enzyme activity (catalase, superoxide dismutase, thioredoxin reductase), which was impaired following UV irradiation. Moreover, ascorbic acid additional stimulated UV-induced bilitranslocase activity responsible for rutin transport, and therefore favored rutin effect on Nrf2 pathway, simultaneously differentiating the reaction of keratinocytes and fibroblasts. In keratinocytes, Nrf2 is strongly activated, while in fibroblasts decreased Nrf2 activity was observed. Used mixture, also significantly silenced UV-induced expression of pro-inflammatory factor NFKB and pro-apoptotic proteins such as caspases 3,8 , and 9 . These results showed that ascorbic acid and rutin are complementary in their antioxidant actions, transport and signaling functions. Their combined antioxidant, antiinflammatory and antiapoptotic actions suggest rutin and ascorbic acid are a potentially cytoprotective team against UV-induced skin damage.
\end{abstract}

Keywords Skin cells $\cdot$ UV radiation $\cdot$ Rutin $\cdot$ Ascorbic acid $\cdot$ Redox balance

\begin{tabular}{|c|c|c|c|c|}
\hline \multicolumn{3}{|c|}{ Abbreviations } & CE & Capillary electrophoresis \\
\hline \multirow{3}{*}{\multicolumn{2}{|c|}{$\begin{array}{l}\text { 4-HNE } \\
\text { 8-isoPGF2 } \alpha \\
\text { ABTS }\end{array}$}} & 4-Hydroxynonenal & $\mathrm{COX}$ & Cyclooxygenase \\
\hline & & 8-Iso-prostaglandin F2 $\alpha$ & $\mathrm{Cu} / \mathrm{Zn}-\mathrm{SOD}$ & Superoxide dismutase \\
\hline & & 2,2'-Azinobis-(3-ethylbenzothiazoline- & DMEM & Dulbecco's modified eagle medium \\
\hline \multicolumn{2}{|c|}{ ABTS } & 6-sulfonic acid) & DMSO & Dimethyl sulfoxide \\
\hline \multirow{2}{*}{\multicolumn{2}{|c|}{$\begin{array}{l}\text { Ascorbic A. } \\
\text { BPE }\end{array}$}} & Ascorbic acid & ELISA & Enzyme-linked immunosorbent assay \\
\hline & & Bovine pituitary extract & ERK & Extracellular signal-regulated kinase \\
\hline \multirow{2}{*}{\multicolumn{3}{|c|}{ Catalase }} & FBS & Fetal bovine serum \\
\hline & & & FRAP & Ferric reducing activity of plasma \\
\hline \multirow{3}{*}{\multicolumn{3}{|c|}{$\begin{array}{l}\text { Electronic supplementary material The online version of this } \\
\text { article (https://doi.org/10.1007/s00403-019-01898-w) contains } \\
\text { supplementary material, which is available to authorized users. }\end{array}$}} & GC & Gas chromatography \\
\hline & & & GSH & Glutathione \\
\hline & & & GSH-Px & Glutathione peroxidase \\
\hline \multirow[t]{2}{*}{$\triangle$} & \multirow{2}{*}{\multicolumn{2}{|c|}{$\begin{array}{l}\text { Agnieszka Gęgotek } \\
\text { agnieszka.gegotek@umb.edu.pl }\end{array}$}} & hEGF & Human epidermal growth factor \\
\hline & & & HPLC & High-performance liquid chromatography \\
\hline & \multirow{2}{*}{\multicolumn{2}{|c|}{$\begin{array}{l}\text { Department of Analytical Chemistry, Medical University } \\
\text { of Bialystok, Mickiewicza 2D, 15-222 Białystok, Poland }\end{array}$}} & IL & Interleukin \\
\hline & & & $\mathrm{LC}$ & Liquid chromatography \\
\hline
\end{tabular}




$\begin{array}{ll}\text { LDL } & \text { Lipoprotein } \\ \text { LOX } & \text { Lipoxygenase } \\ \text { MAPK } & \text { Mitogen-activated protein kinase } \\ \text { MRM } & \text { Multiple reaction monitoring mode } \\ \text { MS } & \text { Mass spectrometry } \\ \text { MTT } & \begin{array}{l}\text { Methylthiazolyldiphenyl-tetrazolium } \\ \text { bromide }\end{array} \\ & \text { Nuclear factor kappa-light-chain-enhancer } \\ \text { NFKB } & \text { of activated B cells } \\ & \text { NADPH oxidase } \\ \text { NOX } & \text { Nuclear factor (erythroid-derived 2)-like 2 } \\ \text { Nrf2 } & \text { Phosphate-buffered saline } \\ \text { PBS } & \text { Reactive oxygen species } \\ \text { ROS } & \text { Selected ion monitoring mode } \\ \text { SIM } & \text { Sulforhodamine B } \\ \text { SRB } & \text { Trolox equivalent antioxidant capacity } \\ \text { TEAC } & \text { Transforming grown factor } \alpha \\ \text { TGF } \alpha & 2,4,6 \text {-Tripyridyl-S-triazine } \\ \text { TPTZ } & \text { Thioredoxin } \\ \text { Trx } & \text { Thioredoxin reductase } \\ \text { TrxR } & \text { Ultrahigh-performance liquid } \\ \text { UPLC } & \text { chromatography } \\ & \text { Xanthine oxidase }\end{array}$

\section{Introduction}

Under physiological conditions, skin cells are characterized by the constant generation of reactive oxygen species (ROS), as well as the well-developed antioxidant system, which maintains the redox balance. The antioxidant system is based on small molecular weight antioxidants including glutathione, vitamins $\mathrm{A}, \mathrm{E}$, and $\mathrm{C}$, as well as antioxidant proteins, which mainly consist of superoxide dismutase, catalase, glutathione peroxidase, thioredoxin, and thioredoxin reductase, which protect cells from ROS-mediated disturbances [31, 42]. Protein antioxidants act to protect skin cells by supporting Nrf2, a ROS-dependent transcription factor, which initiates the transcription of cytoprotective and antioxidant genes [25].

Despite the maintenance of redox balance, frequent exposure of skin cells to the UVA and UVB radiation in natural sunlight stimulates pro-oxidative enzyme activity and impairs the action of antioxidants, resulting in oxidative stress $[7,21]$. Previous studies have shown that exposure to UVA and UVB radiation leads to the activation of many factors involved in mitogen-activated protein (MAP)-dependent signaling kinases, including ERK1/2 and transcription factors dependent on redox potential, e.g., Nrf2 [24, 68]. It has also been suggested that disturbances in the activity of some of these agents, particularly Nrf2, may even lead to neoplastic changes [18]. Moreover, the activation of multiple mechanisms associated with MAP kinases leads to the activation of the NFKB transcription factor and induces an inflammatory reaction $[22,54]$.

Because of disturbed antioxidant abilities, UV radiation induces enhanced ROS-mediated modifications in skin cells, particularly in lipid peroxidation, resulting in increased levels of 4-hydroxynonenal (4-HNE) and prostaglandins that may act as signaling molecules [47, 87]. Prostaglandins are known pro-inflammatory factors, while 4-HNE forms adducts with a number of different proteins which may affect their activity in various ways [47, 67]. As a consequence, the pro-oxidative action of UV radiation leads to metabolic disorders in the skin, including apoptosis, and can significantly deteriorate the condition of the skin [14].

Therefore, there is a constant need for effective skin protection compounds with antioxidant properties, and singlecompound therapies are now being replaced with poly-compound therapies to maximize skin protection. Compounds with different chemical structures usually differ in their properties and when combined, may modify their mutual biological activity, which in many cases leads to synergy. Despite the differences in their chemical structure (Fig. 1), rutin and ascorbic acid both show antioxidant and antiinflammatory effects and can be easily absorbed and transported from both skin surface and blood system [21, 24, 28]. When applied to the skin, ascorbic acid provides significant protection against inflammation and sunburn [3]. Data from the literature indicate that ascorbic acid shows photoprotective potential and can normalize mitochondrial membrane polarization in UVA-irradiated human skin fibroblasts [69]. Moreover, ascorbic acid is essential for collagen biosynthesis in fibroblasts [70]. Despite its antioxidant properties, rutin influences cellular metabolism. Rutin is a flavonoid found in plant extracts, and inhibits the activities of cyclooxygenases and lipoxygenases, thereby reducing pro-inflammatory processes [8]. Moreover, rutin exerts cytoprotective effects on cells exposed to various types of radiation by substantially increasing their viability [79]. So far, the interaction of orally administered rutin with ascorbic acid has been demonstrated in terms of their antiinflammatory and vascular sealing actions $[4,51]$. Moreover, it has been demonstrated that rutin increases the organic reduction ability of ascorbic acid [2]. However, the combined effect of rutin and ascorbic acid on the metabolism of skin cells exposed to UV radiation has been not evaluated yet.

According to the described pro-oxidative effect of UV radiation, as well as relevance of effective stimulation of the antioxidant and antiapoptotic system, the aim of this study was to investigate the combined effect of ascorbic acid and rutin on redox homeostasis, lipid and protein oxidative modifications, and apoptosis of human skin cells (keratinocytes and fibroblasts) after UVA and UVB irradiation. 


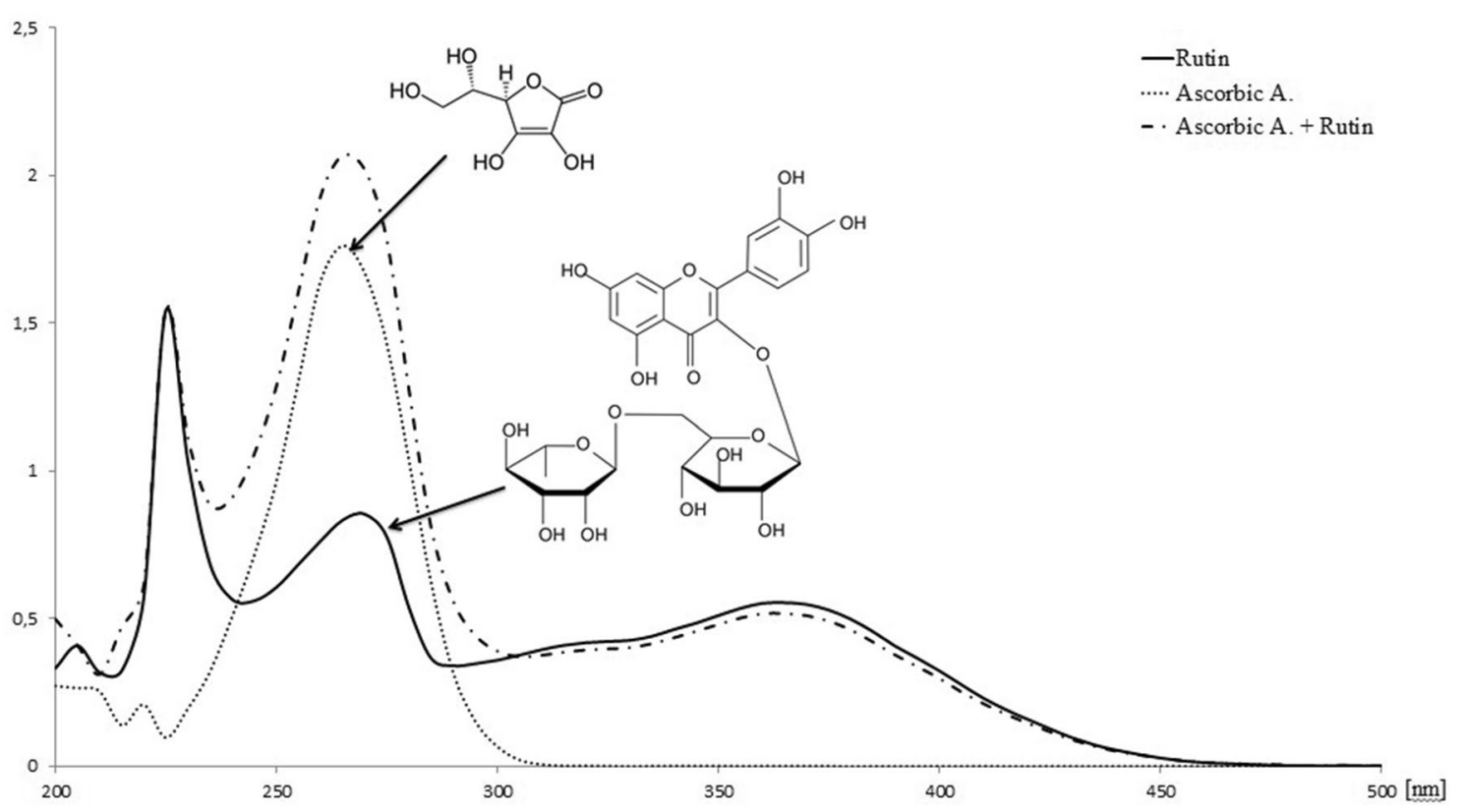

Fig. 1 The chemical structure of rutin and ascorbic acid, and their absorption spectrum

\section{Materials and methods}

\section{Ascorbic acid and rutin antioxidant properties}

\section{TEAC method}

Radical cation $\mathrm{ABTS}^{+}$was prepared according to method of Re et al. [65]. Once the radical was formed, $\mathrm{ABTS}^{+}$was mixed with appropriately diluted ascorbic acid $(100 \mu \mathrm{M})$ or/and rutin $(25 \mu \mathrm{M})$ and the absorbance was measured at $734 \mathrm{~nm}$ after $10 \mathrm{~min}$. Results are expressed vs. antioxidant properties of Trolox.

\section{FRAP method}

The FRAP method was based on that of Benzie and Strain [6]. FRAP reagent consists of: $100 \mathrm{mM}$ acetate buffer, $10 \mathrm{mM}$ TPTZ in $40 \mathrm{mM} \mathrm{HCl}$ and $20 \mathrm{mM}$ ferric (III) chloride. FRAP reagent and the ascorbic acid $(100 \mu \mathrm{M})$ or/and rutin $(25 \mu \mathrm{M})$ were mixed for 4 min and next the absorbance was measured at $593 \mathrm{~nm}$. Results are expressed vs. antioxidant properties of Trolox.

\section{Folin-Ciocalteu method}

Foline-Ciocalteu $(\mathrm{F}-\mathrm{C})$ reagent was mixed with ascorbic acid $(100 \mu \mathrm{M})$ or/and rutin $(25 \mu \mathrm{M})$ and incubated in the

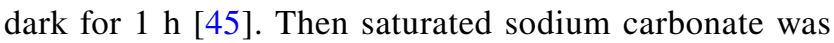

added and again incubated in dark again for $1 \mathrm{~h}$. The absorbance was measured at $764 \mathrm{~nm}$. Results are expressed vs. antioxidant properties of caffeic acid.

\section{Cell culture}

Human skin cell lines used in experiment were obtained from American Type Culture Collection and cultured in a humidified atmosphere of $5 \% \mathrm{CO}_{2}$ at $37{ }^{\circ} \mathrm{C}$. Immortalized keratinocytes (CDD 1102 KERTr) were transformed with human papillomavirus 16 (HPV-16) E6/E7, while fibroblasts (CCD 1112Sk) were isolated from normal foreskin of Caucasian newborn male and used in passage 12 . The growth media for each line were prepared as follows: for keratinocytes-keratinocyte-SFM medium with $1 \%$ Bovine Pituitary Extract (BPE) and human recombinant Epidermal Growth Factor (hEGF); for fibroblasts-Dulbecco's Modified Eagle Medium (DMEM) with 10\% fetal bovine serum (FBS). All media were supplemented with $50 \mu \mathrm{g} / \mathrm{ml}$ streptomycin and $50 \mathrm{U} / \mathrm{ml}$ penicillin. Sterile and cell culture reagents were obtained from Gibco (Grand Island, NY, USA).

When the cells reached $70 \%$ confluence, they were washed with PBS buffer $\left(37^{\circ} \mathrm{C}\right)$ and exposed to UV radiation in cold PBS $\left(4^{\circ} \mathrm{C}\right)$. Radiation doses totaled for keratinocytes were $30 \mathrm{~J} / \mathrm{cm}^{2}$ and $60 \mathrm{~mJ} / \mathrm{cm}^{2}$, and for fibroblasts were $20 \mathrm{~J} / \mathrm{cm}^{2}$ and $200 \mathrm{~mJ} / \mathrm{cm}^{2}$, for UVA and UVB, respectively, using Bio-Link Crosslinker BLX 312/365 (Vilber Lourmat, German). Exposure dose was chosen corresponding to $70 \%$ cell viability measured by the MTT assay [17]. Control cells were incubated in parallel without irradiation. 
To examine the effect of ascorbic acid and rutin on UVradiated skin cells, following cells exposure to UV radiation, they were incubated $24 \mathrm{~h}$ under standard conditions in medium containing $100 \mu \mathrm{M}$ ascorbic acid or/and $25 \mu \mathrm{M}$ rutin in $0.1 \%$ DMSO. The concentration of used compounds was chosen due to the maximum dose that does not change the cell viability measured by the MTT assay [17]. Parallel cells were cultured without irradiation in medium containing above supplements. To maintain the same conditions for all experimental and control groups, all media contained $0.1 \%$ DMSO. The presented data were normalized per milligram of protein. The total protein content in samples was measured using a Bradford assay [9].

\section{Examination of cells viability}

The viability of experimental cells was measured by the sulforhodamine B assay [75]. $24 \mathrm{~h}$ following exposure to UVA/ UVB irradiation and rutin/ascorbic acid treatment, cells were fixed with $10 \%$ trichloroacetic acid and stained with $0.4 \%$ sulforhodamine B in $10 \%$ acetic acid for $30 \mathrm{~min}$. The bound SRB was determined at $540 \mathrm{~nm}$. Cells proliferation was plotted by expressing the absorbance of treated wells as a percentage of control wells.

\section{Examination of transmembrane transport}

\section{Determination of bilitranslocase activity}

The bilitranslocase transport activity was measured spectrophotometrically $[20,59]$. The results were normalized to protein concentration and expressed as units per milligram of protein.

\section{Examination of intracellular ROS generation}

\section{Determination of superoxide anion generation}

The generation of superoxide anions was detected using an electron spin resonance spectrometer e-scan (Noxygen $\mathrm{GmbH} /$ Bruker Biospin GmbH, Germany), where stable nitroxide CM radicals were formed [40]. The generation of superoxide anions was reported in nanomolar concentration per minute and normalized per milligram of protein.

\section{Determination of pro-oxidant enzyme activities}

NADPH oxidase (NOX-EC 1.6.3.1) activity was measured using lucigenin as a luminophore. Enzyme-specific activity was described in RLU (Relative Luminescence Units) per milligram protein [27].

Xanthine oxidase (XO-EC1.17.3.2) activity was estimated by uric acid formation from xanthine through the measurement of the increase in absorbance at $290 \mathrm{~nm}$ [62]. Enzyme-specific activity was described in microunits per milligram of protein.

\section{Estimation of antioxidant capacity}

\section{Determination of antioxidant enzymes activity}

Glutathione peroxidase (GSH-Px-EC.1.11.1.6) activity was assessed spectro-photometrically [58]. Enzyme-specific activity was calculated in milliunits per milligram of protein.

Glutathione reductase (GSSG-R-EC.1.6.4.2) activity was measured by monitoring the oxidation of NADPH at $340 \mathrm{~nm}$ [53]. Enzyme-specific activity was calculated in milliunits per milligram of protein.

Catalase (CAT-EC 1.11.1.9) activity was assayed spectrophotometrically at $240 \mathrm{~nm}$ by the rate of hydrogen peroxide decomposition [1]. Enzyme-specific activity was expressed in milliunits per milligram of protein.

Superoxide dismutase (Cu/Zn-SOD-EC.1.15.1.1) activity was determined according to the method of Misra and Fridovich [52] as modified by Sykes [39]. Enzyme-specific activity was calculated in milliunits per milligram of protein.

The thioredoxin reductase (TrxR-EC. 1.8.1.9) activity was measured using a commercially available kit (SigmaAldrich, St. Louis, MO, USA). Enzyme activity was measured in units per milligram of protein.

\section{Determination of non-enzymatic antioxidant level}

Glutathione was quantified using the capillary electrophoresis (CE) method [49]. Separation was performed on a $47-\mathrm{cm}$ capillary and $50 \mathrm{~m}$ i.d. and was operated at $27 \mathrm{kV}$ with UV detection at $200 \pm 10 \mathrm{~nm}$. The GSH concentration was determined using a calibration curve range of $1-120 \mathrm{nmol} / \mathrm{mL}\left(r^{2}\right.$ -0.9985 ) and normalized for milligrams of protein.

High-performance liquid chromatography (HPLC) was used to detect the levels of vitamins C [34] and vitamin E [85]. For determination of vitamin C, separation was performed using RP-18 column and UV detection at $250 \mathrm{~nm}$. Vitamin E was extracted from the cell lysates using hexane, diluted in ethanol, and injected onto the RP-18 column. UV detection at $294 \mathrm{~nm}$ was applied. The concentration of vitamins was determined using a calibration curve range $1.25-20 \mu \mathrm{g} / \mathrm{mL}\left(r^{2}=0.9999\right)$ for vitamin $\mathrm{C}$, and $5-25 \mathrm{mg} / \mathrm{L}$ $\left(r^{2}=0.9999\right)$ for vitamin $\mathrm{E}$ and normalized for milligrams of protein.

The rutin level was measured using an Agilent 1290 HPLC system [20]. The mobile phase consisted of water containing $0.1 \%(\mathrm{v} / \mathrm{v})$ TFA with linear gradients of $5 \%$ acetonitrile at $t=0-20 \%$ acetonitrile at $5 \mathrm{~min}$, followed by an increase to $30 \%$ acetonitrile at $10 \mathrm{~min}$. Finally, $90 \%$ acetonitrile was used from $18 \mathrm{~min}$ onward for $5 \mathrm{~min}$. Detection was 
carried out on a diode array detector. The presented data are based on detection at $360 \mathrm{~nm}$ (the absorption maximum of rutin). The concentration of rutin was determined using a calibration curve range: $1-15 \mu \mathrm{M} / \mathrm{mL}\left(r^{2}-0.9998\right)$ and normalized for milligrams of protein.

Thioredoxin level was quantified using ELISAs [46]. Proteins were incubated at $4{ }^{\circ} \mathrm{C}$ overnight with anti-thioredoxin primary antibody (Abcam, Cambridge, MA, USA). After incubation with peroxidase blocking solution $\left(3 \% \mathrm{H}_{2} \mathrm{O}_{2}, 3 \%\right.$ fat free dry milk), goat anti-rabbit secondary antibody solution (Dako, Carpinteria, CA, USA) was added. Following $1 \mathrm{~h}$ incubation, chromogen substrate solution $\left(0.1 \mathrm{mg} \mathrm{mL}^{-1}\right.$ TMB, $0.012 \% \mathrm{H}_{2} \mathrm{O}_{2}$ ) was added. Spectral absorption was read at $450 \mathrm{~nm}$ with the reference filter set to $620 \mathrm{~nm}$. The thioredoxin concentration was determined using a calibration curve range of $0.5-20 \mu \mathrm{g}\left(r^{2}-0.9983\right)$ and was normalized for milligrams of protein.

\section{Estimation of lipid peroxidation}

8-Iso-prostaglandin F2 $\alpha$ (8-isoPGF2 $\alpha$ ) was assayed by the modified LC-MS method of Coolen [13, 24] using an Agilent 1290 UPLC system interfaced with an Agilent 6460 triple quadrupole mass spectrometer. 8 -isoPGF $2 \alpha$ was analyzed in negative-ion mode using MRM mode: $\mathrm{m} / \mathrm{z}$ $353.2 \rightarrow 193.1$ (for 8 -isoPGF2 $\alpha$ ) and $357.2 \rightarrow 197.1$ (for 8 -isoPGF $2 \alpha$-d 4 ). Obtained results were normalized for milligrams of protein.

4-Hydroxynonenal was measured by GC/MS in selected ion monitoring (SIM) mode, as the O-PFB-oxime-TMS derivatives using benzaldehyde-D6 as an internal standard [48]. The ions used were: $\mathrm{m} / \mathrm{z} 333.0$ and 181.0 for 4-HNEPFB-TMS and $m / z 307.0$ for IS derivatives. Obtained results were normalized for milligrams of protein.

\section{Examination of protein modifications}

\section{Determination of structure modification}

Protein oxidative modifications were estimated according to the level of carbonyl groups. Carbonyl group level was determined spectrophotometrically $(370 \mathrm{~nm})$ using 2,4-dinitrophenylhydrazine [41], and described in nanomolar per milligram of protein.

\section{Examination of protein expression}

Western blot analysis was performed according to Eissa and Seada [16]. Cell lysates were separated by $10 \%$ Tris-Glycine SDS-PAGE and transferred into nitrocellulose membrane. Primary antibodies against Keap1 (host: goat), cytochrome $c$ (host: mouse) (Santa Cruz Biotechnology, CA, USA); caspase 9, KAP1, p62, Bach1, HO-1 (host: rabbit), Bax, p53, Bcl-2, NFkB (p52), caspase 8 (host: mouse) (SigmaAldrich, St. Louis, MO, USA); caspase 3 (host: goat) (R\&D, MN, USA), and phospho-Nrf2 (Ser40) (host: rabbit) (Bioss Antibodies Inc., MA, USA) were used at a concentration of $1: 1000$. As an internal loading control, $\beta$-actin (host: mouse) (Sigma-Aldrich, St. Louis, MO, USA) was used. Following incubation, membranes were incubated with alkaline phosphatase-conjugated secondary antibodies against rabbit, mouse or goat (host: goat or rabbit) (Sigma-Aldrich, St. Louis, MO, USA) at a concentration of 1:2000. Protein bands were visualized using the BCIP/NBT liquid substrate system (Sigma-Aldrich, St. Louis, MO, USA) and quantitated using the Versa Doc System and Quantity One software (Bio-Rad Laboratories Inc., CA, USA). The results are expressed as a percentage of the control cells results.

\section{Statistical analysis}

Data were analyzed using standard statistical analyses, including multivariate analysis (one-way ANOVA), and the results are expressed as the mean \pm standard deviation (SD) for $n=3$. $p$ values less than 0.05 were considered significant.

\section{Results}

There was no change in the absorption spectra of ascorbic acid or rutin when the compounds were combined together (Fig. 1), suggesting that neither compound modifies the chemical structure of the other.

\section{Antioxidant properties of ascorbic acid and rutin}

Ascorbic acid (Ascorbic A.) showed three times higher antioxidant properties than rutin (Rutin), measured by the cation radical scavenging activity by the ferric-reducing activity of plasma (FRAP) test (Fig. 2). However, the mixture of ascorbic acid and rutin (Ascorbic A. + Rutin) had approximately $20 \%$ higher antioxidant properties compared to Ascorbic A alone. The F-C test showed that Ascorbic A. + Rutin acted two times stronger than Ascorbic A. or Rutin alone.

\section{Ascorbic acid and rutin effect on cell viability}

Ascorbic acid and rutin, used in selected concentrations separately, as well as jointly, partially prevented a UV-induced decrease in the viability of fibroblasts and keratinocytes (Fig. 3). The effect of Ascorbic A. + Rutin on keratinocytes was evident even under standard conditions, resulting in a $25 \%$ increase in keratinocyte viability. Individually, Ascorbic A. and Rutin each increased the viability of UVA-irradiated keratinocytes by about 15\%; however, the combination of Ascorbic A. + Rutin improved the viability of UVA- and 

of rutin $(25 \mu \mathrm{M})$ and ascorbic acid $(100 \mu \mathrm{M})$ measured by the ABST, FRAP and F-C tests. Mean values \pm SD of three independent experiments are

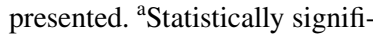
cant differences vs. Ascorbic A., $p<0.05$; ${ }^{\text {b Statistically }}$ significant differences vs. Rutin, $p<0.05$
Fig. 2 Antioxidant properties

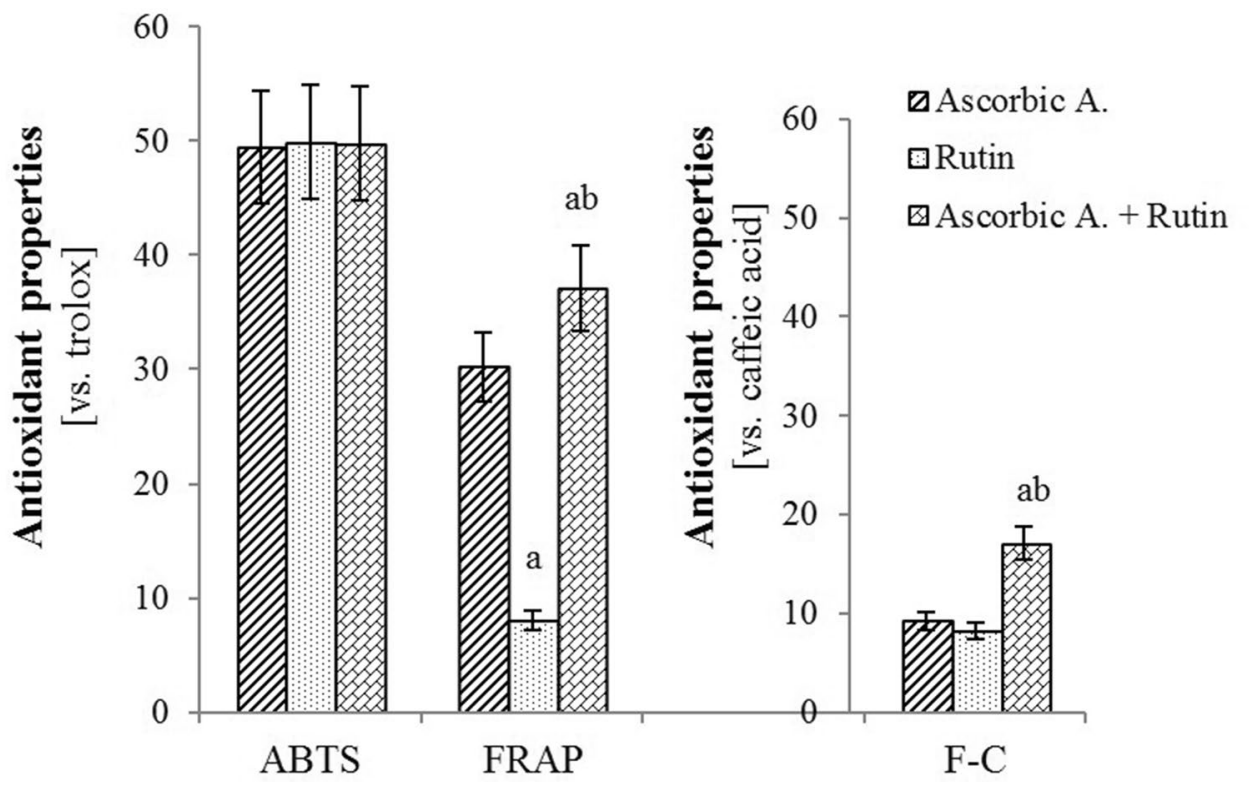

Fig. 3 The viability of keratinocytes and fibroblasts exposed to UVA $\left(30 \mathrm{~J} / \mathrm{cm}^{2}\right.$ and $20 \mathrm{~J} / \mathrm{cm}^{2}$, respectively) and UVB irradiation $\left(60 \mathrm{~mJ} / \mathrm{cm}^{2}\right.$ and $200 \mathrm{~mJ} /$ $\mathrm{cm}^{2}$, respectively) and treated with rutin $(25 \mu \mathrm{M})$ and ascorbic acid $(100 \mu \mathrm{M})$, measured by the sulforhodamine B assay

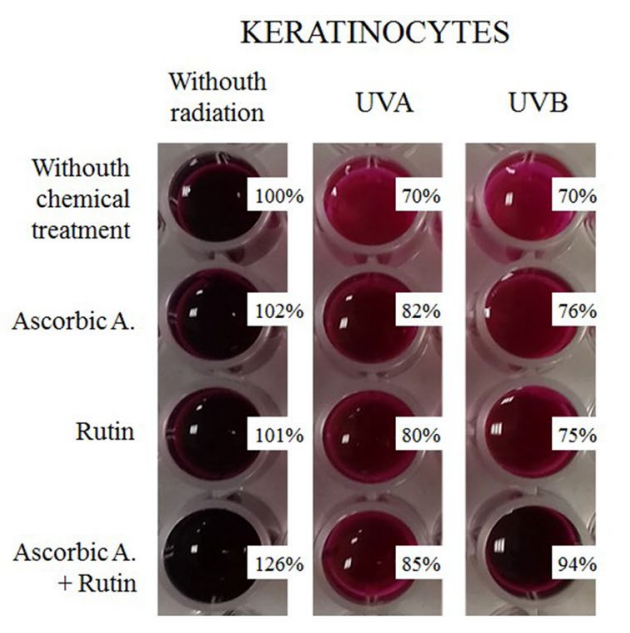

\section{FIBROBLASTS}

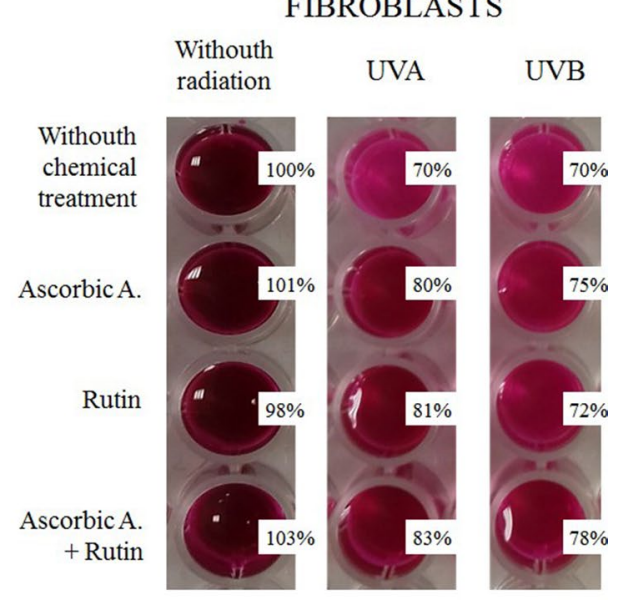

UVB-irradiated keratinocytes by $20 \%$ and $35 \%$, respectively. Both supplements alone and in combination did not have a significant effect on the viability of fibroblasts under standard conditions; however, Ascorbic A. + Rutin increased the viability of UVA- and UVB-irradiated fibroblasts by approximately $15 \%$ and $10 \%$, respectively.

\section{The effect of ascorbic acid and rutin on transmembrane transport}

The activity of the transmembrane transporter, bilitranslocase, following Rutin treatment in UV-irradiated keratinocytes and fibroblasts was enhanced by approximately $30 \%$, while Ascorbic A. did not show any effect. However, Ascorbic A. + Rutin mildly enhances bilitranslocase activity in both cell types compared to non-treated cells (Fig. 4). These changes were accompanied even by two times increase in the rutin level in keratinocytes and fibroblasts treated with Ascorbic A. + Rutin compared with only Rutin-treated cells (Table 1).

\section{The effect of ascorbic acid and rutin on ROS generation}

Ascorbic acid as well as rutin had a strong cytoprotective effect against UVB-induced changes in superoxide anion generation and oxidase activity in both cell types studied (Fig. 5). Ascorbic A.+ Rutin led to a decrease in superoxide anion generation in UVB-irradiated keratinocytes and fibroblasts by about $60 \%$ and $80 \%$, respectively, which was $30 \%$ and $10 \%$ more than in the case of Rutin and Ascorbic A used alone. Moreover, the activity of xanthine oxidase in UV-irradiated keratinocytes was decreased by $50-60 \%$ in all supplemented cells, while NADPH oxidase activity in 


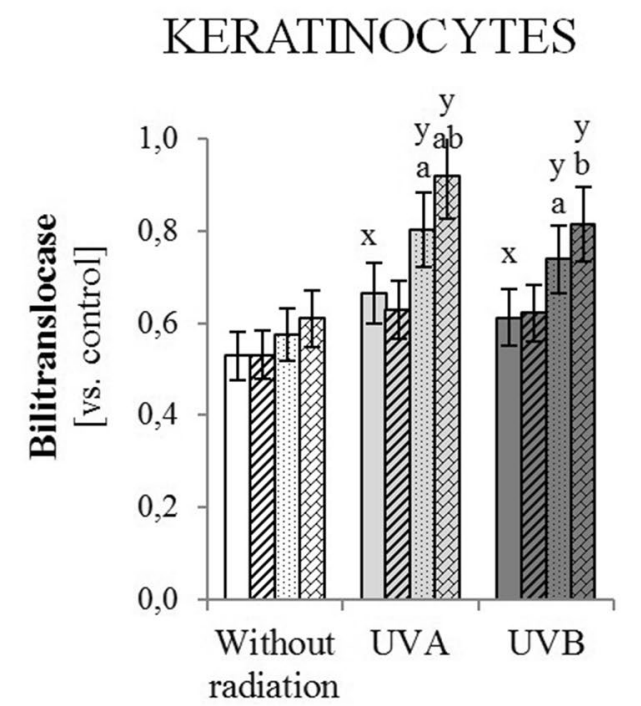

Fig. 4 Bilitranslocase activity in keratinocytes and fibroblasts exposed to UVA ( $30 \mathrm{~J} / \mathrm{cm}^{2}$ and $20 \mathrm{~J} / \mathrm{cm}^{2}$, respectively) and UVB irradiation $\left(60 \mathrm{~mJ} / \mathrm{cm}^{2}\right.$ and $200 \mathrm{~mJ} / \mathrm{cm}^{2}$, respectively) and treated with rutin $(25 \mu \mathrm{M})$ and ascorbic acid $(100 \mu \mathrm{M})$. Mean values \pm SD of three independent experiments are presented. ${ }^{x}$ Statistically significant dif-

\section{FIBROBLASTS}

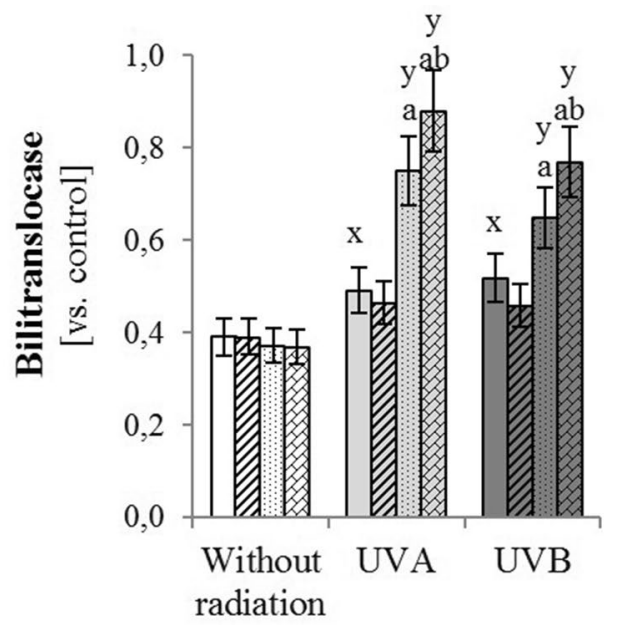

ferences vs. non-treated group, $p<0.05$; ${ }^{\mathrm{y}}$ Statistically significant differences vs. respectively group without chemical treatment, $p<0.05$; ${ }^{a}$ Statistically significant differences vs. Ascorbic A. treated group, $p<0.05$; 'Statistically significant differences vs. Rutin treated group, $p<0.05$

Table 1 Non-enzymatic antioxidant level in keratinocytes and fibroblasts exposed to UVA $\left(30 \mathrm{~J} / \mathrm{cm}^{2}\right.$ and $20 \mathrm{~J} / \mathrm{cm}^{2}$, respectively) and UVB irradiation $\left(60 \mathrm{~mJ} / \mathrm{cm}^{2}\right.$ and $200 \mathrm{~mJ} / \mathrm{cm}^{2}$, respectively) and treated with rutin $(25 \mu \mathrm{M})$ and ascorbic acid $(100 \mu \mathrm{M})$

\begin{tabular}{|c|c|c|c|c|c|c|c|}
\hline & \multirow[t]{2}{*}{ Treatment } & \multicolumn{3}{|l|}{ Keratinocytes } & \multicolumn{3}{|l|}{ Fibroblast } \\
\hline & & - & UVA & UVB & - & UVA & UVB \\
\hline \multirow[t]{4}{*}{ GSH (nmol/mg protein) } & - & $17.1 \pm 0.8$ & $11.5 \pm 0.5^{\mathrm{x}}$ & $10.9 \pm 0.5^{x}$ & $13.7 \pm 0.8$ & $8.7 \pm 0.5^{x}$ & $8.1 \pm 0.4^{\mathrm{x}}$ \\
\hline & Ascorbic A. & $18.6 \pm 0.9$ & $16.1 \pm 0.8^{y}$ & $14.6 \pm 0.7^{y}$ & $12.3 \pm 0.7$ & $9.7 \pm 0.5$ & $8.7 \pm 0.5$ \\
\hline & Rutin & $17.8 \pm 0.8$ & $15.4 \pm 0.7^{y}$ & $13.5 \pm 0.6^{\mathrm{y}}$ & $14.3 \pm 0.8^{\mathrm{a}}$ & $10.7 \pm 0.6^{\mathrm{y}}$ & $10.7 \pm 0.5^{\mathrm{y}, \mathrm{a}}$ \\
\hline & Ascorbic A. + Rutin & $19.5 \pm 0.9^{\mathrm{y}, \mathrm{a}, \mathrm{b}}$ & $16.4 \pm 0.8^{y}$ & $14.8 \pm 0.7^{y}$ & $12.1 \pm 0.7^{y, b}$ & $13.6 \pm 1.0^{\mathrm{y}, \mathrm{a}, \mathrm{b}}$ & $10.7 \pm 0.6^{\mathrm{y}, \mathrm{a}}$ \\
\hline \multirow[t]{4}{*}{ Vit E ( $\mu \mathrm{g} / \mathrm{mg}$ protein) } & - & $5.2 \pm 0.3$ & $4.9 \pm 0.4$ & $3.9 \pm 0.2^{x}$ & $4.0 \pm 0.2$ & $2.4 \pm 0.1^{\mathrm{x}}$ & $3.3 \pm 0.2^{\mathrm{x}}$ \\
\hline & Ascorbic A. & $5.9 \pm 0.2^{y}$ & $5.0 \pm 0.2$ & $4.7 \pm 0.2^{y}$ & $4.1 \pm 0.2$ & $2.5 \pm 0.1$ & $3.4 \pm 0.2$ \\
\hline & Rutin & $5.1 \pm 0.2^{\mathrm{a}}$ & $5.0 \pm 0.2$ & $4.5 \pm 0.2^{\mathrm{y}}$ & $4.4 \pm 0.2$ & $3.5 \pm 0.2^{\mathrm{y}, \mathrm{a}}$ & $3.4 \pm 0.2$ \\
\hline & Ascorbic A. + Rutin & $6.1 \pm 0.3^{\mathrm{y}, \mathrm{a}, \mathrm{b}}$ & $5.1 \pm 0.2$ & $5.5 \pm 0.3^{\mathrm{y}, \mathrm{a}, \mathrm{b}}$ & $4.8 \pm 0.2^{\mathrm{y}, \mathrm{a}, \mathrm{b}}$ & $2.9 \pm 0.1^{\mathrm{y}, \mathrm{a}, \mathrm{b}}$ & $3.9 \pm 0.2^{\mathrm{y}, \mathrm{a}, \mathrm{b}}$ \\
\hline \multirow[t]{4}{*}{ Vit $C$ ( $\mu \mathrm{g} / \mathrm{mg}$ protein) } & - & n.d. & n.d. & n.d. & $6.9 \pm 0.4$ & $5.2 \pm 0.3^{\mathrm{x}}$ & $5.8 \pm 0.3$ \\
\hline & Ascorbic A. & $24.6 \pm 1.4$ & $24.9 \pm 1.2$ & $21.8 \pm 1.0$ & $26.6 \pm 1.5^{\mathrm{y}}$ & $24.5 \pm 1.4^{y}$ & $23.9 \pm 1.4^{y}$ \\
\hline & Rutin & n.d. & n.d. & n.d. & $7.1 \pm 0.4^{\mathrm{a}}$ & $5.5 \pm 0.3^{\mathrm{a}}$ & $5.9 \pm 0.3^{\mathrm{a}}$ \\
\hline & Ascorbic A. + Rutin & $29.5 \pm 1.4^{\mathrm{a}}$ & $28.8 \pm 1.3^{\mathrm{a}}$ & $26.3 \pm 1.2^{\mathrm{a}}$ & $35.8 \pm 1.5^{\mathrm{y}, \mathrm{a}, \mathrm{b}}$ & $32.4 \pm 1.3^{\mathrm{y}, \mathrm{a}, \mathrm{b}}$ & $35.4 \pm 1.5^{\mathrm{y}, \mathrm{a}, \mathrm{b}}$ \\
\hline \multirow[t]{4}{*}{ Rutin $(\mu \mathrm{M} / \mathrm{mg}$ protein) } & - & n.d. & n.d. & n.d. & n.d. & n.d. & n.d. \\
\hline & Ascorbic A. & n.d. & n.d. & n.d. & n.d. & n.d. & n.d. \\
\hline & Rutin & $3.4 \pm 0.2$ & $9.7 \pm 0.6$ & $8.1 \pm 0.5$ & $1.2 \pm 0.1$ & $8.9 \pm 0.4$ & $4.7 \pm 0.2$ \\
\hline & Ascorbic A. + Rutin & $6.2 \pm 0.4^{b}$ & $11.4 \pm 0.8^{b}$ & $11.7 \pm 0.7^{b}$ & $3.7 \pm 0.2^{\mathrm{b}}$ & $10.1 \pm 0.5^{\mathrm{b}}$ & $6.8 \pm 0.3^{b}$ \\
\hline \multirow[t]{4}{*}{$\operatorname{Trx}(\mu \mathrm{g} / \mathrm{mg}$ protein $)$} & - & $1.61 \pm 0.08$ & $1.01 \pm 0.05^{\mathrm{x}}$ & $0.83 \pm 0.04^{\mathrm{x}}$ & $1.31 \pm 0.07$ & $1.13 \pm 0.06^{\mathrm{x}}$ & $0.91 \pm 0.05^{\mathrm{x}}$ \\
\hline & Ascorbic A. & $1.84 \pm 0.07^{\mathrm{y}}$ & $1.23 \pm 0.06^{\mathrm{y}}$ & $1.03 \pm 0.05^{\mathrm{y}}$ & $1.41 \pm 0.08$ & $1.22 \pm 0.07$ & $1.33 \pm 0.07^{\mathrm{y}}$ \\
\hline & Rutin & $1.51 \pm 0.07^{\mathrm{a}}$ & $1.22 \pm 0.06^{\mathrm{y}}$ & $1.13 \pm 0.05^{\mathrm{y}, \mathrm{a}}$ & $1.32 \pm 0.07$ & $1.14 \pm 0.06$ & $1.22 \pm 0.06^{\mathrm{y}}$ \\
\hline & Ascorbic A. + Rutin & $1.51 \pm 0.07^{\mathrm{a}}$ & $1.32 \pm 0.06^{\mathrm{y}}$ & $1.24 \pm 0.06^{\mathrm{y}, \mathrm{a}, \mathrm{b}}$ & $1.51 \pm 0.08^{\mathrm{y}, \mathrm{b}}$ & $1.32 \pm 0.08^{\mathrm{y}, \mathrm{b}}$ & $1.31 \pm 0.07^{\mathrm{y}, \mathrm{b}}$ \\
\hline
\end{tabular}

Mean values \pm SD of three independent experiments are presented

${ }^{\mathrm{x}}$ Statistically significant differences vs. non-treated group, $p<0.05$

${ }^{\mathrm{y}}$ Statistically significant differences vs. respectively group without chemical treatment, $p<0.05$

${ }^{a}$ Statistically significant differences vs. Ascorbic A. treated group, $p<0.05$

${ }^{\mathrm{b}}$ Statistically significant differences vs. Rutin treated group, $p<0.05$ 
Fig. 5 Xanthine oxidase and NADPH oxidase activity, as well as intracellular superoxide anion generation in keratinocytes and fibroblasts exposed to UVA $\left(30 \mathrm{~J} / \mathrm{cm}^{2}\right.$ and $20 \mathrm{~J} / \mathrm{cm}^{2}$, respectively) and UVB irradiation $\left(60 \mathrm{~mJ} / \mathrm{cm}^{2}\right.$ and $200 \mathrm{~mJ} /$ $\mathrm{cm}^{2}$, respectively) and treated with rutin $(25 \mu \mathrm{M})$ and ascorbic acid $(100 \mu \mathrm{M})$. Mean values \pm SD of three independent experiments are presented. ${ }^{\mathrm{x}} \mathrm{Sta}$ tistically significant differences vs. non-treated group, $p<0.05$; ${ }^{\mathrm{y}}$ Statistically significant differences vs. respectively group without chemical treatment,

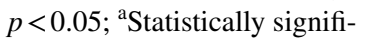
cant differences vs. Ascorbic A. treated group, $p<0.05$; ${ }^{\text {b Statisti- }}$ cally significant differences vs. Rutin-treated group, $p<0.05$
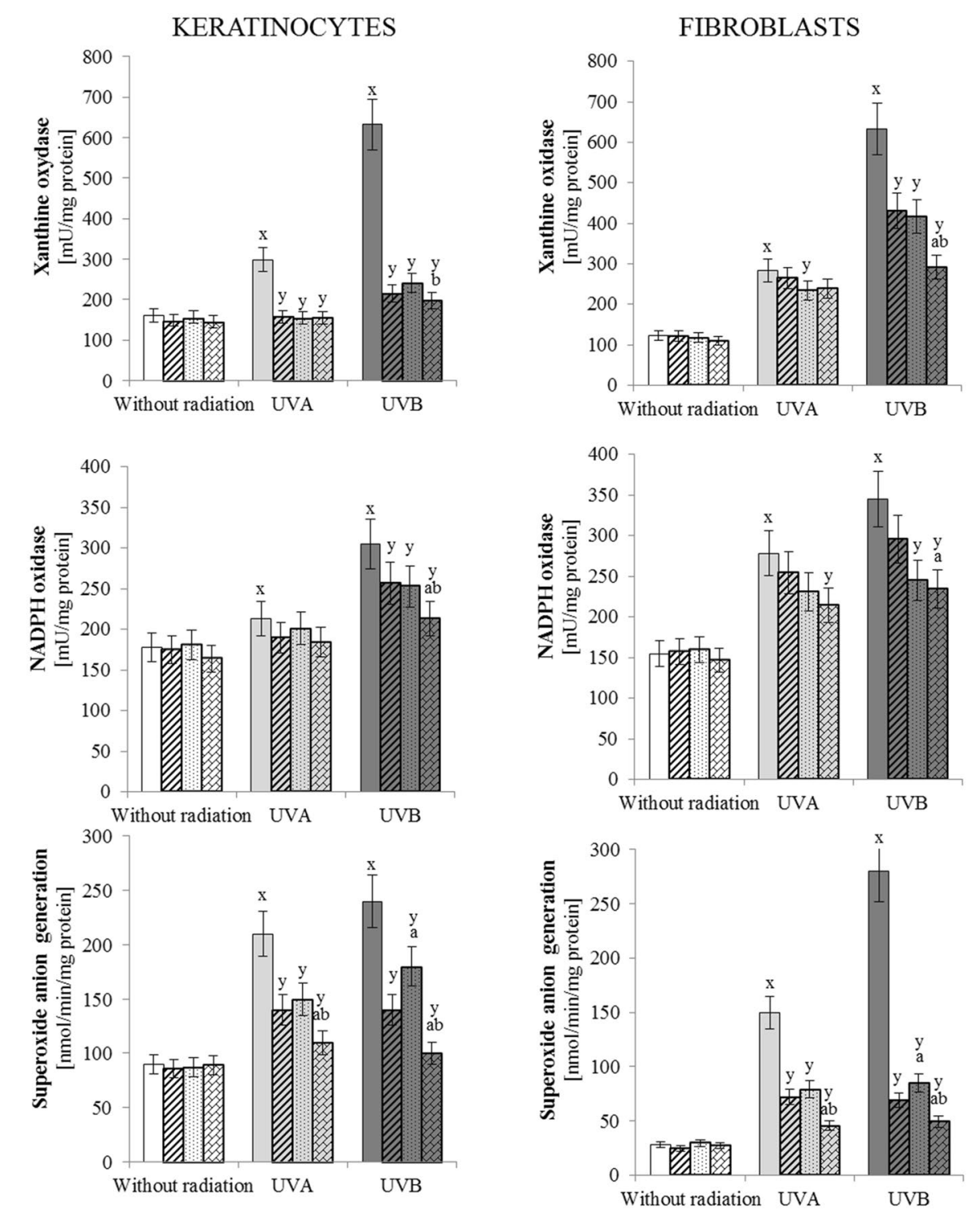

Without chemical treatment
Ascorbic A.
固Rutin 因Ascorbic A. + Rutin
UVB-irradiated keratinocytes was decreased by $15 \%$ following Rutin or Ascorbic A. treatment, and by $30 \%$ following Ascorbic A. + Rutin treatment. Simultaneously, in UVBirradiated fibroblasts, Ascorbic A. or Rutin treatment led to a decrease in xanthine oxidase by approximately $30 \%$, while Ascorbic A. + Rutin caused a decrease by about $50 \%$. In addition, the activity of NADPH oxidase was decreased by Ascorbic A. + Rutin treatment by $30 \%$ in both UVA- and UVB-irradiated fibroblasts.

\section{The effect of ascorbic acid and rutin on antioxidant enzyme activity}

The effect of ascorbic acid and rutin treatment on antioxidant enzymes activity in UV-irradiated skin cells is shown in Table 2. In all UV-irradiated cells, ascorbic acid and rutin treatment led to decrease in UV-enhanced glutathione peroxidase (GSH-Px) activity, while in the case of GSSG-R the same effect was observed only in UVA- and UVB-irradiated keratinocytes, and UVA-irradiated fibroblasts. Moreover, Ascorbic A. + Rutin had a significantly stronger effect than either Rutin or Ascorbic A. alone on thioredoxin reductase ( $\operatorname{TrxR}$ ) and catalase (CAT) activity. In UVA- and UVB-irradiated keratinocytes, TrxR and CAT activity increased by about $50 \%$ and twofold, respectively, and for fibroblasts TrxR and CAT activity increased by $55 \%$ and threefold following UVA radiation, and twofold and around $30 \%$ following UVB radiation. Also in the case of SOD activity Ascorbic A. + Rutin significantly prevented a UVB-induced decrease in keratinocytes (by $10 \%$ ) and in fibroblasts (twofold increase compared to UVB-irradiated cells). 
Table 2 Antioxidant enzyme activity in keratinocytes and fibroblasts exposed to UVA ( $30 \mathrm{~J} / \mathrm{cm}^{2}$ and $20 \mathrm{~J} / \mathrm{cm}^{2}$, respectively) and UVB irradiation $\left(60 \mathrm{~mJ} / \mathrm{cm}^{2}\right.$ and $200 \mathrm{~mJ} / \mathrm{cm}^{2}$, respectively) and treated with rutin $(25 \mu \mathrm{M})$ and ascorbic acid $(100 \mu \mathrm{M})$

\begin{tabular}{|c|c|c|c|c|c|c|c|}
\hline & \multirow[t]{2}{*}{ Treatment } & \multicolumn{3}{|l|}{ Keratinocytes } & \multicolumn{3}{|l|}{ Fibroblast } \\
\hline & & - & UVA & UVB & - & UVA & UVB \\
\hline \multirow[t]{4}{*}{ GSH-Px activity (mU/mg protein) } & - & $14.6 \pm 0.7$ & $34.4 \pm 1.7^{\mathrm{x}}$ & $37.5 \pm 1.9^{x}$ & $11.8 \pm 0.6$ & $26.3 \pm 1.5^{\mathrm{x}}$ & $30.5 \pm 1.8^{\mathrm{x}}$ \\
\hline & Ascorbic A. & $12.5 \pm 0.6^{\mathrm{y}}$ & $24.3 \pm 1.2^{\mathrm{y}}$ & $24.8 \pm 1.2^{\mathrm{y}}$ & $7.3 \pm 0.4^{\mathrm{y}}$ & $15.6 \pm 0.9^{y}$ & $18.3 \pm 1.1^{\mathrm{y}}$ \\
\hline & Rutin & $12.3 \pm 0.6^{y}$ & $28.3 \pm 1.4^{\mathrm{y}, \mathrm{a}}$ & $25.6 \pm 1.3^{\mathrm{y}}$ & $9.1 \pm 0.5^{\mathrm{y}, \mathrm{a}}$ & $12.6 \pm 0.7^{\mathrm{y}, \mathrm{a}}$ & $20.7 \pm 1.1^{\mathrm{y}, \mathrm{a}}$ \\
\hline & Ascorbic A. + Rutin & $13.4 \pm 0.7$ & $24.1 \pm 1.2^{\mathrm{y}, \mathrm{b}}$ & $24.6 \pm 1.2^{\mathrm{y}}$ & $9.4 \pm 0.6^{\mathrm{y}, \mathrm{a}}$ & $10.1 \pm 1.2^{\mathrm{y}, \mathrm{a}, \mathrm{b}}$ & $19.5 \pm 1.2^{y}$ \\
\hline \multirow[t]{4}{*}{ GSSG-R activity (mU/mg protein) } & - & $10.2 \pm 0.5$ & $15.6 \pm 0.7^{\mathrm{x}}$ & $16.3 \pm 0.8^{\mathrm{x}}$ & $24.1 \pm 1.4$ & $47.7 \pm 2.8^{\mathrm{x}}$ & $63.8 \pm 3.7^{\mathrm{x}}$ \\
\hline & Ascorbic A. & $11.4 \pm 0.6$ & $12.1 \pm 0.6^{\mathrm{y}}$ & $13.2 \pm 0.7^{\mathrm{y}}$ & $27.7 \pm 1.6^{\mathrm{y}}$ & $43.9 \pm 2.6^{\mathrm{y}}$ & $64.1 \pm 3.8$ \\
\hline & Rutin & $12.4 \pm 0.6^{\mathrm{y}}$ & $12.4 \pm 0.6^{y}$ & $14.3 \pm 0.7^{y}$ & $31.2 \pm 1.8^{\mathrm{y}, \mathrm{a}}$ & $40.2 \pm 2.4^{\mathrm{y}, \mathrm{a}}$ & $64.3 \pm 3.3$ \\
\hline & Ascorbic A. + Rutin & $11.6 \pm 0.6$ & $12 \pm 0.6^{\mathrm{y}}$ & $12.3 \pm 0.6^{\mathrm{y}, \mathrm{b}}$ & $29.4 \pm 1.7^{y}$ & $42.1 \pm 2.5^{\mathrm{y}}$ & $60.3 \pm 3.5$ \\
\hline \multirow[t]{4}{*}{ CAT activity (mU/mg protein) } & - & $9.9 \pm 0.5$ & $6.1 \pm 0.3^{\mathrm{x}}$ & $6.3 \pm 0.3^{x}$ & $8.4 \pm 0.5$ & $9.3 \pm 0.5$ & $11.3 \pm 0.7^{\mathrm{x}}$ \\
\hline & Ascorbic A. & $13.5 \pm 0.7^{y}$ & $12.6 \pm 0.6^{y}$ & $10.3 \pm 0.5^{\mathrm{y}}$ & $7.7 \pm 0.4$ & $7.9 \pm 0.5$ & $8.9 \pm 0.5^{\mathrm{y}}$ \\
\hline & Rutin & $11.0 \pm 0.6^{\mathrm{y}, \mathrm{a}}$ & $7.5 \pm 0.4^{\mathrm{y}, \mathrm{a}}$ & $7.5 \pm 0.3^{\mathrm{y}, \mathrm{a}}$ & $9.1 \pm 0.5^{\mathrm{y}, \mathrm{a}}$ & $8.8 \pm 0.5$ & $10.9 \pm 0.5^{\mathrm{y}, \mathrm{a}}$ \\
\hline & Ascorbic A. + Rutin & $14.5 \pm 0.7^{\mathrm{y}, \mathrm{b}}$ & $12.9 \pm 0.6^{\mathrm{y}, \mathrm{b}}$ & $12.4 \pm 0.6^{\mathrm{y}, \mathrm{a}, \mathrm{b}}$ & $10.7 \pm 0.6^{\mathrm{y}, \mathrm{a}}$ & $23.9 \pm 1.4^{\mathrm{y}, \mathrm{a}, \mathrm{b}}$ & $15.5 \pm 0.9^{\mathrm{y}, \mathrm{a}, \mathrm{b}}$ \\
\hline \multirow[t]{4}{*}{ SOD activity (mU/mg protein) } & - & $28.0 \pm 1.4$ & $24.0 \pm 1.2^{\mathrm{x}}$ & $23.0 \pm 1.2^{\mathrm{x}}$ & $25.5 \pm 1.5$ & $21.2 \pm 1.3^{\mathrm{x}}$ & $13.8 \pm 0.8^{x}$ \\
\hline & Ascorbic A. & $27.8 \pm 1.4$ & $25.9 \pm 1.3$ & $25.3 \pm 1.3$ & $26.1 \pm 1.5$ & $23.1 \pm 1.4$ & $20.7 \pm 1.2^{\mathrm{y}}$ \\
\hline & Rutin & $28.0 \pm 1.4$ & $25.1 \pm 1.3$ & $24.1 \pm 1.2$ & $27.9 \pm 1.6$ & $24.1 \pm 1.4$ & $21.2 \pm 1.1^{\mathrm{y}}$ \\
\hline & Ascorbic A. + Rutin & $28.1 \pm 1.4$ & $25.8 \pm 1.3$ & $25.8 \pm 1.3^{\mathrm{y}}$ & $28.1 \pm 1.6$ & $20.5 \pm 1.2^{\mathrm{b}}$ & $26.7 \pm 1.6^{\mathrm{y}, \mathrm{a}, \mathrm{b}}$ \\
\hline \multirow[t]{4}{*}{ TrxR (U/mg protein) } & - & $10.5 \pm 0.5$ & $9.1 \pm 0.4^{\mathrm{x}}$ & $8.3 \pm 0.4^{\mathrm{x}}$ & $12.8 \pm 0.8$ & $10.2 \pm 0.6^{\mathrm{x}}$ & $7.7 \pm 0.4^{\mathrm{x}}$ \\
\hline & Ascorbic A. & $12.6 \pm 0.6^{\mathrm{y}}$ & $12.4 \pm 0.6^{y}$ & $10.4 \pm 0.5^{\mathrm{y}}$ & $12.9 \pm 0.8$ & $12.8 \pm 0.4^{\mathrm{y}}$ & $9.7 \pm 0.5^{\mathrm{y}}$ \\
\hline & Rutin & $10.4 \pm 0.5^{\mathrm{a}}$ & $10.3 \pm 0.5^{\mathrm{a}}$ & $9.5 \pm 0.5$ & $12.9 \pm 0.8$ & $13.1 \pm 0.8^{\mathrm{y}}$ & $10.2 \pm 0.5^{\mathrm{y}}$ \\
\hline & Ascorbic A. + Rutin & $13.5 \pm 0.7^{y, b}$ & $12.7 \pm 0.6^{\mathrm{y}, \mathrm{b}}$ & $11.0 \pm 0.6^{\mathrm{y}, \mathrm{b}}$ & $12.5 \pm 0.6$ & $15.6 \pm 0.7^{\mathrm{y}, \mathrm{a}, \mathrm{b}}$ & $14.1 \pm 0.7^{\mathrm{y}, \mathrm{a}, \mathrm{b}}$ \\
\hline
\end{tabular}

Mean values \pm SD of three independent experiments are presented

${ }^{\mathrm{x}}$ Statistically significant differences vs. non-treated group, $p<0.05$

${ }^{y}$ Statistically significant differences vs. respectively group without chemical treatment, $p<0.05$

${ }^{\text {a }}$ Statistically significant differences vs. Ascorbic A. treated group, $p<0.05$

${ }^{\mathrm{b}}$ Statistically significant differences vs. Rutin treated group, $p<0.05$

\section{The effect of ascorbic acid and rutin on non-enzymatic antioxidant level}

The results indicated that non-enzymatic antioxidant levels in UV-irradiated cells were more susceptible to the Ascorbic A. + Rutin treatment than Ascorbic A. or Rutin alone (Table 1). One of the most sensitive antioxidants was thioredoxin, which decreased level in UV-irradiated cells was restored even by $45-50 \%$ in the case of UVB-irradiated keratinocytes and fibroblasts or by $30 \%$ and $15 \%$ in UVAirradiated cells. Similar changes were observed in the case of vitamins $\mathrm{E}$ and $\mathrm{C}$, where Ascorbic A. + Rutin treatment significantly increased vitamin $\mathrm{E}$ levels in UVB-irradiated keratinocytes by $40 \%$, and in UVA- and UVB-irradiated fibroblast by $25 \%$ and $20 \%$ compared to UVA- and UVBirradiated cells, respectively. In the case of vitamin $\mathrm{C}$ level, Ascorbic A. + Rutin treatment led to a 15-20\% increase compared to UV-irradiated keratinocytes treated only with Ascorbic A., and a 35-50\% increase compared to UV-irradiated and Ascorbic A. treated fibroblasts. However, only UVirradiated fibroblasts showed a stronger effect to Ascorbic
A. + Rutin treatment than Ascorbic A. or Rutin in the case of GSH level, which was increased by $55 \%$ and $25 \%$ following UVA and UVB radiation, respectively.

\section{The effect of ascorbic acid and rutin on the Nrf2 pathway}

Ascorbic A. + Rutin influenced the Nrf2 pathway in various skin cell lines (Fig. 6). A twofold increase in the level of phosphor-Nrf2, as well as in its main target heme oxygenase-1 (HO-1) was observed in UV-irradiated keratinocytes following Ascorbic A. + Rutin treatment. These changes were accompanied by a $50 \%$ increase in the expression of the Nrf2 inhibitor, Keap1, which was the opposite of the action of Rutin itself. Moreover, following UV radiation, Ascorbic A. + Rutin treatment prevented changes induced by Ascorbic A. or Rutin in the level of Bach1 and KAP1, while p62 expression was decreased by $10 \%$. In the case of UV-irradiated fibroblasts, Ascorbic A. + Rutin led to the decrease in phosphor-Nrf2 level by $40 \%$ and by $10-25 \%$ in HO-1 level. Moreover, the levels of Nrf2 inhibitors, Keap1 

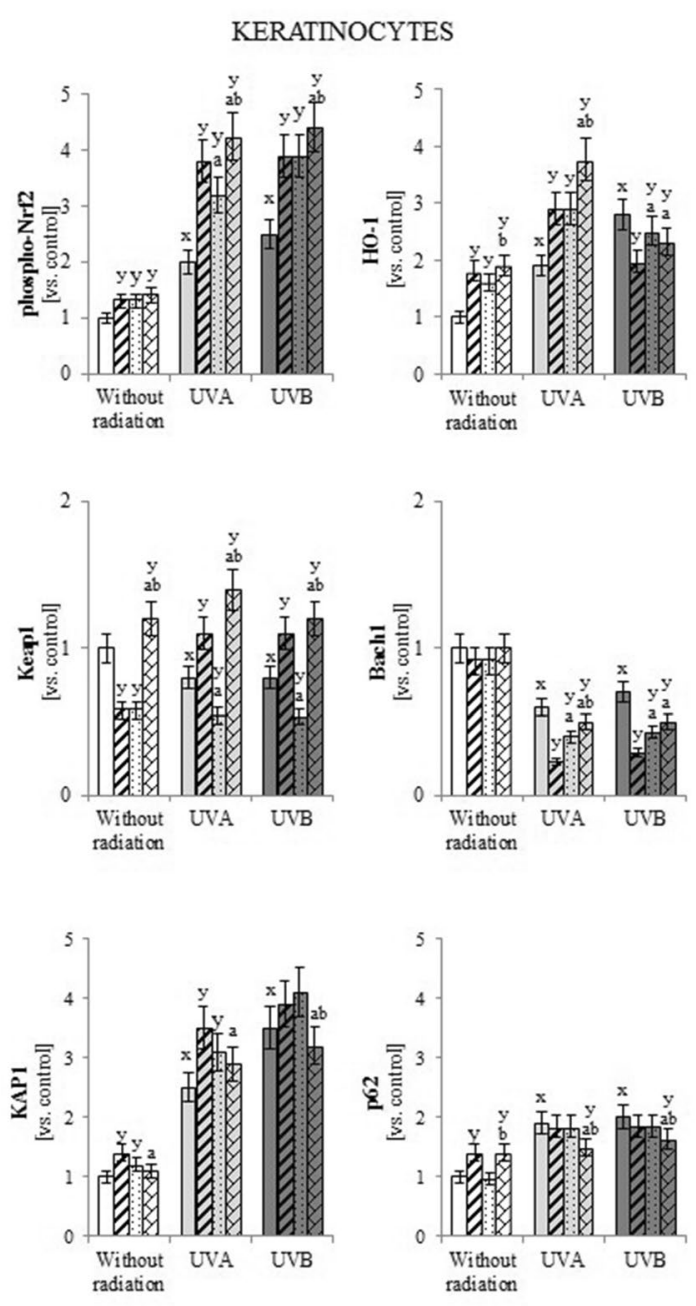

$\square$ Without chemical treatment

$\square$ Ascorbic A.

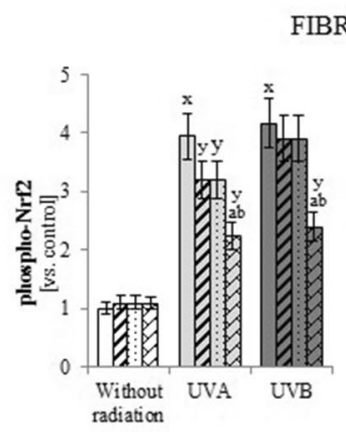

IBROBLASTS
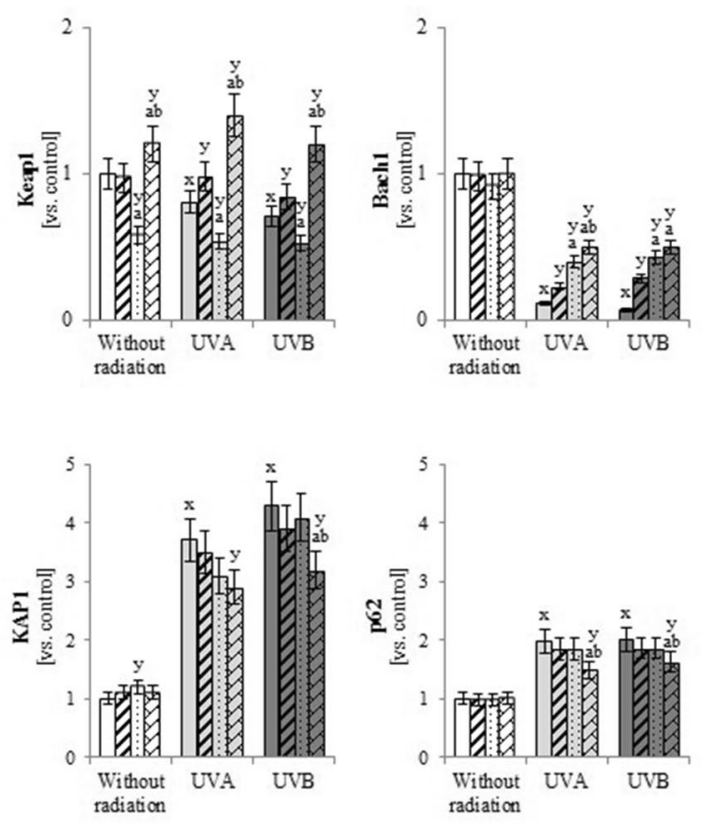

$\square$ Rutin

$\triangle$ Ascorbic A. + Rutin
Fig. 6 The level of phospho-Nrf2 (Ser40) transcription factor, its activators (KAP1, p62) and inhibitors (Keap1, Bach1), and Nrf2 main target- $\mathrm{HO}-1$ in keratinocytes and fibroblasts exposed to UVA $\left(30 \mathrm{~J} / \mathrm{cm}^{2}\right.$ and $20 \mathrm{~J} / \mathrm{cm}^{2}$, respectively) and UVB irradiation $(60 \mathrm{~mJ} /$ $\mathrm{cm}^{2}$ and $200 \mathrm{~mJ} / \mathrm{cm}^{2}$, respectively) and treated with rutin $(25 \mu \mathrm{M})$ and ascorbic acid $(100 \mu \mathrm{M})$. Electrophorogram images are included

and Bach1, in UV-irradiated fibroblasts following Ascorbic A. + Rutin treatment were increased two and five times, respectively, while the expression of Nrf2 inhibitors, KAP1 and p62, were decreased by approximately $20 \%$.

\section{The effect of ascorbic acid and rutin on the modification of cellular components}

The cytoprotective effect of ascorbic acid and rutin on cellular components is shown in Table 3. Ascorbic A. + Rutin revealed a stronger effect than Ascorbic A. or Rutin alone on the decrease in the level of 8-isoPGF $\alpha$ in UVA- and UVBirradiated keratinocytes (by $15 \%$ and $40 \%$, respectively). A as a supplementary material. Mean values \pm SD of three independent experiments are presented. ${ }^{\mathrm{x}}$ Statistically significant differences vs. non-treated group, $p<0.05$; ${ }^{\mathrm{y}}$ Statistically significant differences vs. respectively group without chemical treatment, $p<0.05$; ${ }^{\text {a Statistically }}$ significant differences vs. Ascorbic A. treated group, $p<0.05$; ${ }^{\mathrm{b}}$ Statistically significant differences vs. Rutin treated group, $p<0.05$

similar effect, but to a lesser extent, was observed in UVA- and UVB-irradiated fibroblasts, where the level of 8-isoPGF $\alpha$ was decreased following Ascorbic A. + Rutin treatment by $20 \%$ and $10 \%$, respectively. Moreover, in the case of protein oxidation, Ascorbic A. + Rutin had a stronger effect than Ascorbic A. or Rutin in preventing carbonyl group formation in keratinocytes and fibroblasts exposed to UV radiation.

\section{The effect of ascorbic acid and rutin on inflammation and apoptosis}

The effect of ascorbic acid and rutin on inflammatory processes and apoptosis in UV-irradiated cells is shown in 
Table 3 The level of lipids and proteins oxidative modification in keratinocytes and fibroblasts exposed to UVA $\left(30 \mathrm{~J} / \mathrm{cm}^{2} \mathrm{and} 20 \mathrm{~J} / \mathrm{cm}^{2}\right.$, respectively) and UVB irradiation $\left(60 \mathrm{~mJ} / \mathrm{cm}^{2}\right.$ and $200 \mathrm{~mJ} / \mathrm{cm}^{2}$, respectively) and treated with rutin $(25 \mu \mathrm{M})$ and ascorbic acid $(100 \mu \mathrm{M})$

\begin{tabular}{|c|c|c|c|c|c|c|c|}
\hline & \multirow[t]{2}{*}{ Treatment } & \multicolumn{3}{|c|}{ Keratinocytes } & \multicolumn{3}{|l|}{ Fibroblast } \\
\hline & & - & UVA & UVB & - & UVA & UVB \\
\hline \multirow[t]{4}{*}{ 8-isoPFG2a (pg/mg protein) } & - & $1.4 \pm 0.07$ & $2.4 \pm 0.12^{\mathrm{x}}$ & $3.5 \pm 0.17^{\mathrm{x}}$ & $6.2 \pm 0.3$ & $13.8 \pm 0.8^{\mathrm{x}}$ & $15.4 \pm 0.9^{x}$ \\
\hline & Ascorbic A. & $1.2 \pm 0.06^{\mathrm{y}}$ & $2.2 \pm 0.11$ & $2.5 \pm 0.12^{y}$ & $5.8 \pm 0.3$ & $11.2 \pm 0.6^{\mathrm{y}}$ & $15.2 \pm 0.8$ \\
\hline & Rutin & $1.6 \pm 0.08^{\mathrm{a}}$ & $2.3 \pm 0.11$ & $2.8 \pm 0.14^{\mathrm{y}, \mathrm{a}}$ & $7.8 \pm 0.4^{\mathrm{y}, \mathrm{a}}$ & $10.1 \pm 0.5^{y}$ & $14.2 \pm 0.7$ \\
\hline & Ascorbic A. + Rutin & $1.5 \pm 0.07^{\mathrm{a}}$ & $2.0 \pm 0.10^{\mathrm{y}, \mathrm{a}, \mathrm{b}}$ & $2.1 \pm 0.10^{y, a, b}$ & $5.6 \pm 0.3^{\mathrm{b}}$ & $11.3 \pm 0.6^{\mathrm{y}, \mathrm{b}}$ & $13.4 \pm 0.7^{\mathrm{y}, \mathrm{a}}$ \\
\hline \multirow[t]{4}{*}{ 4-HNE (nmol/mg protein) } & - & $26.1 \pm 1.3$ & $73.4 \pm 3.6^{\mathrm{x}}$ & $52.4 \pm 2.6^{\mathrm{x}}$ & $54.2 \pm 3.1$ & $87.1 \pm 5.1^{\mathrm{x}}$ & $71.3 \pm 4.2^{\mathrm{x}}$ \\
\hline & Ascorbic A & $29.6 \pm 1.4^{y}$ & $64.3 \pm 3.2^{\mathrm{y}}$ & $48.3 \pm 2.4^{y}$ & $55.7 \pm 3.2$ & $77.3 \pm 4.5^{\mathrm{y}}$ & $61.3 \pm 4.2^{y}$ \\
\hline & Rutin & $27.3 \pm 1.3^{\mathrm{a}}$ & $59.5 \pm 2.9^{\mathrm{y}, \mathrm{a}}$ & $50.6 \pm 2.5$ & $50.9 \pm 3.0$ & $61.9 \pm 3.0^{\mathrm{y}, \mathrm{a}}$ & $58.7 \pm 2.9^{y}$ \\
\hline & Ascorbic A. + Rutin & $29.1 \pm 1.4^{\mathrm{y}}$ & $57.1 \pm 2.8^{\mathrm{y}, \mathrm{a}}$ & $45.6 \pm 2.2^{\mathrm{y}, \mathrm{a}, \mathrm{b}}$ & $52.5 \pm 3.0$ & $62.2 \pm 4.2^{\mathrm{y}, \mathrm{a}}$ & $55.3 \pm 3.8^{\mathrm{y}}$ \\
\hline \multirow[t]{4}{*}{ Carbonyl groups (U/mg protein) } & - & $0.41 \pm 0.01$ & $0.53 \pm 0.02^{\mathrm{x}}$ & $0.54 \pm 0.02^{\mathrm{x}}$ & $0.31 \pm 0.02$ & $0.52 \pm 0.03^{x}$ & $0.71 \pm 0.03^{\mathrm{x}}$ \\
\hline & Ascorbic A & $0.42 \pm 0.02$ & $0.48 \pm 0.02^{y}$ & $0.47 \pm 0.02^{\mathrm{y}}$ & $0.34 \pm 0.02$ & $0.41 \pm 0.02^{\mathrm{y}}$ & $0.54 \pm 0.03^{y}$ \\
\hline & Rutin & $0.45 \pm 0.02$ & $0.47 \pm 0.02^{y}$ & $0.45 \pm 0.02^{\mathrm{y}}$ & $0.32 \pm 0.02$ & $0.48 \pm 0.02^{\mathrm{a}}$ & $0.55 \pm 0.02^{\mathrm{y}}$ \\
\hline & Ascorbic A. + Rutin & $0.43 \pm 0.02$ & $0.44 \pm 0.02^{\mathrm{a}}$ & $0.35 \pm 0.01^{\mathrm{y}, \mathrm{a}, \mathrm{b}}$ & $0.34 \pm 0.02$ & $0.34 \pm 0.06^{\mathrm{y}, \mathrm{a}, \mathrm{b}}$ & $0.36 \pm 0.02^{\mathrm{y}, \mathrm{a}, \mathrm{b}}$ \\
\hline
\end{tabular}

Mean values \pm SD of three independent experiments are presented

${ }^{\mathrm{x}}$ Statistically significant differences vs. non-treated group, $p<0.05$

${ }^{\mathrm{y}}$ Statistically significant differences vs. respectively group without chemical treatment, $p<0.05$

${ }^{a}$ Statistically significant differences vs. Ascorbic A. treated group, $p<0.05$

${ }^{\mathrm{b}}$ Statistically significant differences vs. Rutin treated group, $p<0.05$

Fig. 7. Ascorbic A. + Rutin partially prevented UV-induced pro-inflammatory factor NFKB expression, however, in a lesser extent than in the case of Ascorbic A. or Rutin used separately. In the case of UV-irradiated fibroblasts, the opposite situation was observed, when Ascorbic A. + Rutin resulted in a stronger decrease in $\mathrm{NF \kappa B}$ expression than Ascorbic A. or Rutin used separately.

Moreover, Ascorbic A. + Rutin had a similar effect as Ascorbic A. on enhanced antiapoptotic protein Bax expression, as well as p53 and Bcl-2 which control the cell cycle in UV-irradiated keratinocytes, simultaneously Ascorbic A. + Rutin prevented increase in the level of cytochrome $c$ in these cells. On the other hand, in UV-irradiated fibroblasts, Ascorbic A. + Rutin decreased the level of Bax and was more effective than Ascorbic A. or Rutin in preventing a UV-induced increase in the levels of p53, cytochrome $c$, and caspases 8 and 9. In the case of Bcl-2 expression, Ascorbic A. + Rutin also significantly enhanced the level of this protein in UVA- and UVB-irradiated fibroblasts.

\section{Discussion}

The main function of the skin is to protect the organism against harmful exogenous factors, and to receive signals from the surrounding environment. UV radiation that reaches the skin cells mainly consists of UVA that penetrates both the epidermis and dermis, and UVB, which is mainly scattered in the outer epidermal layers, and only a small percentage reaches dermis [7]. Therefore, various layers of skin cells reaction to changes in the external environment, which affects not only the condition and appearance of the skin, but also the functioning of the whole organism [15]. The external localization of skin keratinocytes and fibroblasts means that they are mostly exposed to UV radiation from the sunlight, which modifies their specific functions in different ways.

Used in this study mixture of ascorbic acid with rutin is applying in oral formulation for the prevention of colds and flu. Moreover, such formulations are also active in the case of blood vessel enhancement; their use is recommended for vascular diseases and disorders, where rutin decreases the permeability of capillary walls, strengthens them, improves the condition of their wall tension, and prevents edema [19]. Moreover, ascorbic acid is a proline and lysine hydroxylases cofactor, and is essential compound for collagen biosynthesis in skin fibroblasts [11]. In the case of keratinocytes, ascorbic acid is an important factor for the initiation of keratinization, and thus is also required for the maintenance of the natural rate of formation of an outer protective layer of corneocytes [81]. However, the combined effect of ascorbic acid and rutin in skin cells protection against $\mathrm{UV}$ radiation has been not previously been investigated.

\section{Component uptake and skin cell viability}

This study shows that when used together, the tested compounds prevent a UV-induced reduction in cell survival. 


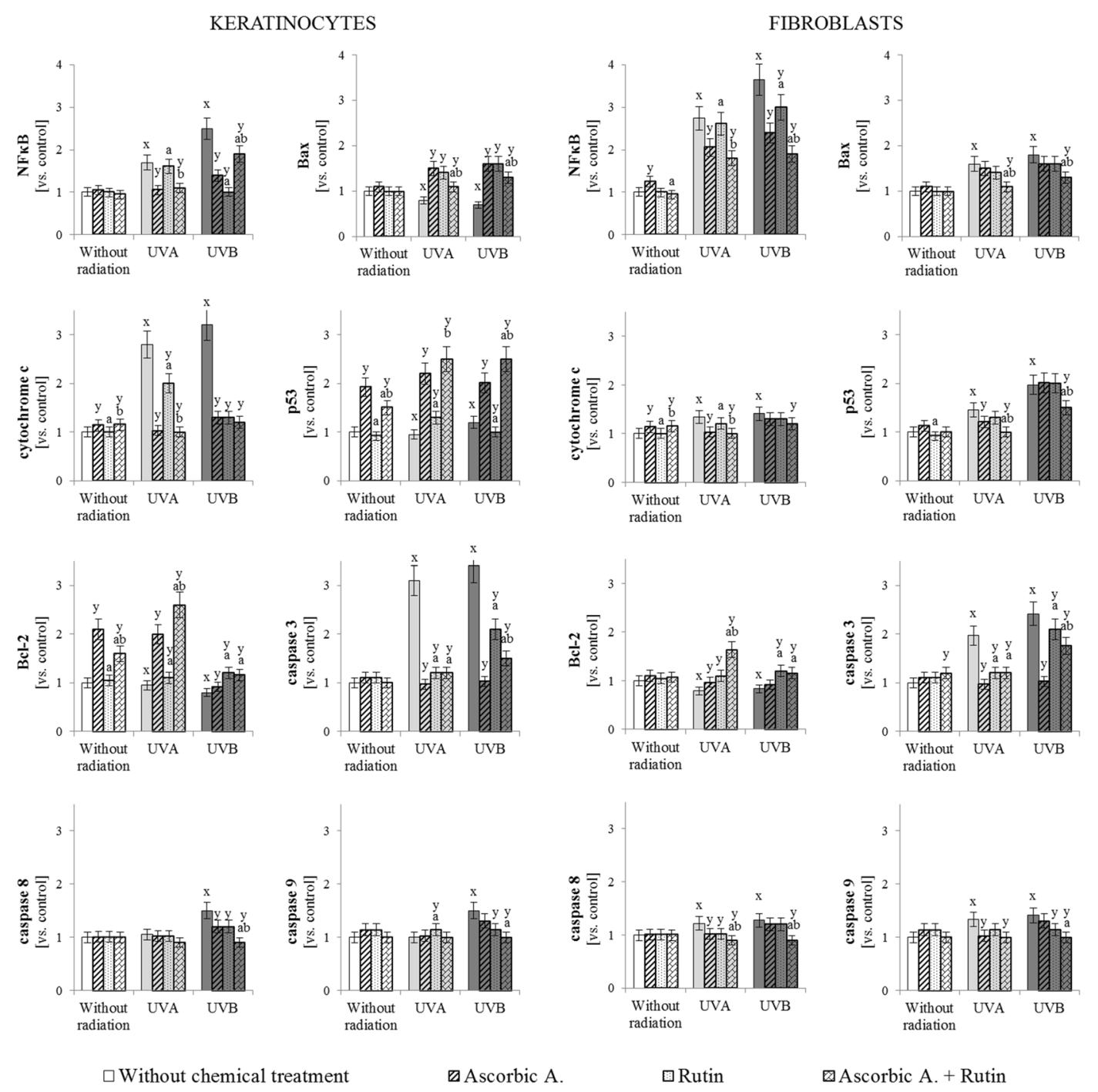

Fig. 7 The level of pro-inflammatory factor NFkB (p52), and proand antiapoptotic proteins (cytochrome $c, \mathrm{p} 53, \mathrm{Bcl}-2$, Caspase 3, 8, 9, Bax) in keratinocytes and fibroblasts exposed to UVA $\left(30 \mathrm{~J} / \mathrm{cm}^{2}\right.$ and $20 \mathrm{~J} / \mathrm{cm}^{2}$, respectively) and UVB irradiation $\left(60 \mathrm{~mJ} / \mathrm{cm}^{2}\right.$ and $200 \mathrm{~mJ} /$ $\mathrm{cm}^{2}$, respectively) and treated with rutin $(25 \mu \mathrm{M})$ and ascorbic acid $(100 \mu \mathrm{M})$. Electrophorogram images are included as a supplementary

material. Mean values \pm SD of three independent experiments are presented. ${ }^{x}$ Statistically significant differences vs. non-treated group, $p<0.05$; ${ }^{\mathrm{y}}$ Statistically significant differences vs. respectively group without chemical treatment, $p<0.05$; ${ }^{a}$ Statistically significant differences vs. Ascorbic A. treated group, $p<0.05$; ${ }^{\text {b }}$ Statistically significant differences vs. Rutin treated group, $p<0.05$

This may be related to the degree to which the tested compounds penetrated into cells, which is associated with the activity of specific transmembrane transporters. Under physiological conditions, rutin is only slightly taken up into the cytosol by bilitranslocase, however, UV radiation favors its transporter activity [20]. Bilitranslocase transports flavonoids according to their concentration [12], and its activity is unaffected by the concentrations of ascorbic acid which were used in this work. However, this study demonstrates that when used in combination, ascorbic acid supports the uptake of rutin, thereby increasing its action [29], what is also shown in this study. The cytoprotective effect of ascorbic acid is mainly related to its antioxidant action in fibroblasts, while in keratinocytes ascorbic acid is a stimulator of cell proliferation and differentiation [21, 71]. On the other hand, the cytoprotective effect of rutin on UV-treated fibroblasts is based on the coordination of fibroblast proliferation, and depending on the location of the cells, rutin may stimulate or inhibit their proliferation [30, 33, 44]. Moreover, rutin can replace the use of ascorbic acid in the culture medium to maintain cell viability of isolated secondary follicles [43]. In addition, rutin promotes wound healing, not only by stimulation of fibroblast proliferation, but also by supporting extracellular matrix production including collagen biosynthesis what is not observed in the case of keratinocytes [78, 84]. The 
application of ascorbic acid and rutin together enhances the cytoprotective action of these compounds.

\section{Intracellular ROS generation}

Enhanced ROS generation after UV radiation threatens the proper functioning of skin cells, however, the structures of ascorbic acid and rutin are rich in hydroxyl groups (Fig. 1) which are conducive to their ability to capture ROS and transform them into less reactive forms $[26,66]$. This, therefore, prevents oxidative modifications of cellular components, as shown by a decrease in the activity of NADPH and xanthine oxidases as well as ROS generation in this study. In the concentrations used in this study, rutin and ascorbic acid have a strong radical scavenger rate, as shown in the previous studies [60]. In addition, earlier studies have shown that the interaction of rutin with ascorbic acid leads to an increase in the reducing properties of ascorbic acid, which is expressed by a decrease in xanthine oxidase activity, responsible for the cytosol production of superoxide anion [74]. In addition, the combined action of rutin and ascorbic acid was more effective than single compound action in the ROS decrease in the animal tissues [77].

\section{Antioxidant capacity}

Due to constant exposure to harmful factors, skin cells are characterized by a well-developed antioxidant system, accompanied by extensive signaling pathways. However, the action of most of the enzymatic and non-enzymatic components of this system is disrupted as a result of skin exposition to UV radiation [22, 50]. Ascorbic acid and rutin support the cellular antioxidant system both as free radical scavengers and by stimulating antioxidant action. Ascorbic acid is the main natural water-soluble antioxidant. It is transported unobstructed through the skin from topical applications or oral doses [10]. In cells, it is known that ascorbic acid cooperates with glutathione in maintaining the reduced form of tocopherol [86], a natural phospholipid membrane protector whose levels are reduced by UV radiation. Because glutathione levels which were decreased by UV radiation are enhanced by the common rutin and ascorbic acid action, and because rutin promotes ascorbic acid action in tocopherol reduction, rutin and ascorbic acid cooperation may have a borderline importance in the protection of membrane lipids against oxidative modifications [57]. GSH is a cofactor of GSH-Px, which is responsible also for lipid protection against peroxidation; however, the compounds used here partially inhibit GSH-Px activity. Moreover, UV radiation decreases the thioredoxin reductase activity as well as thioredoxin level, thereby lowering the effectiveness of thioredoxin-dependent antioxidant system, and exposing the thiol groups of the oxidized proteins to less effective reduction. However, exogenous compounds may activate thioredoxin reductase under oxidative conditions [77], and ascorbic acid and rutin restore the thioredoxindependent system, which is especially important in repairing UV-induced protein modifications. On the other hand, the polyphenolic structure of rutin provides the ability to maintain the redox balance in fibroblasts, which has been already described elsewhere [24]. Additionally, the rutin-induced stimulation of antioxidant enzymes, e.g., SOD or CAT, increased the antioxidant capacity of brain tissue [5]. The antioxidant cooperation of ascorbic acid and rutin that has been indicated in this study was also showed in other studies, where the mixture of rutin and ascorbic acid resulted in a significant increase in the activity of antioxidant enzymes such as SOD or CAT in mammalian kidneys [35].

Reactions of the skin cells to UV-induced oxidative stress are also related to the activation of intracellular and extracellular signaling pathways, leading to changes in transcription factor activity. The transcription factor, Nrf2, is responsible for the expression of genes encoding antioxidant and antiapoptotic proteins [39]. However, the results of this show that ascorbic acid and rutin have the opposite effect in the case of UV-irradiated keratinocytes and fibroblasts. The antioxidant properties of the used in these experiment compounds promote a decrease of the pro-oxidant parameters, therefore the activity of Nrf2 in fibroblasts is also diminished [82], especially following combined application. However, in the case of keratinocytes, which are epidermal cells that are more sensitive, more proliferative and are characterized by increased adaptability, antioxidant compounds additionally increase the activity of Nrf2, which prepares cells for possibly stronger stress [38]. Moreover, it has been also shown that ascorbic acid in skin keratinocytes affects intracellular signaling through protein kinase $\mathrm{C}$ activation [71], which additionally activates Nrf2.

Ascorbic acid and rutin cause also different changes in Keap1 levels. Decrease in Keap1 levels following rutin treatment might be connected with rutin-Keap1 adduct formation, which has been found in UV-irradiated skin fibroblasts [23]. Keap 1 adduct formation changes the conformation of Keap1, leading to the Keap1-Nrf2 dissociation, as well as leading to Keap1 ubiquitination and degradation [37]. However, the interaction of rutin with Keap1 is almost completely abolished in the treatment with ascorbic acid.

\section{Modification of intracellular compounds}

The high energy doses carried by UV radiation lead to disorders of cellular redox balance, and in consequence, lead to oxidative modifications of lipids, nucleic acids, and proteins, which interfere with cellular metabolism by initiating photoreaction processes [22, 32]. These oxidative changes may even result in oncogenic transformation [64]. 
Lipid modifications are particularly important for intra- and extracellular signaling. A mixture of ascorbic acid and rutin prevents increased lipid peroxidation of the skin cells after exposure to UVA and UVB radiation. The reduction of the level of lipid peroxidation products, such as MDA, by ascorbic acid in UV-irradiated keratinocytes has previously been shown [63]. Moreover, use of a rutin and ascorbic acid mixture more effectively prevented the UV radiation-induced oxidation of low density lipoprotein (LDL) and their cytotoxic effects than either ascorbic acid or rutin used separately [55]. On the other hand, it has been shown that ascorbic acid, depending on its growing concentration, affects the condition of membrane lipids and increases or inhibits the penetration of rutin by the micellar lipid bilayer [29, 55].

Regardless of the effect on lipids, the mixture of rutin and ascorbic acid, reduced the generation of ROS and promoted fewer protein modifications. These studies showed that their co-operation prevented an increase in the number of carbonyl groups following UV radiation, and in consequence, cellular proteins did not lose their function and did not impair cellular metabolism. However, rutin is capable of creating adducts with histidine residues in the active site of tyrosinase, which leads to tyrosinase inhibition and stops the production of melanin [74]. Therefore, rutin has the potential to be a potent antipigment agent.

\section{Inflammation}

Both of the antioxidant compounds in this study also exhibit a selective antiinflammatory effect. It has been shown that ascorbic acid is able to downregulate IL-1 $\alpha$ mRNA expression in both UVA-irradiated and non-irradiated cells, while IL-6 mRNA expression remained unaffected [83]. In the case of UVB-irradiated keratinocytes, ascorbic acid downregulated IL-8 mRNA expression [36]. It has also been reported that rutin treatment of neutrophils inhibits the activity of enzymes responsible for the metabolism of fatty acids, cyclooxygenase (COX) and lipoxygenase (LOX), thereby reducing pro-inflammatory factors amount [8, 24], what in this study was observed as a significant decrease in 8-isoPGF2 $\alpha$ level. Moreover, rutin suppresses NFкB activation and down-regulates gene expression of most proinflammatory cytokines/enzymes, except for a significant up-regulation of IL-8 observed under stimulation with TGF $\alpha$ [61]. Combination of these ascorbic acid and rutin activities in UV-irradiated fibroblasts and keratinocytes results in the inhibition of inflammatory processes, as shown by deceased NFkB expression.

\section{Apoptosis}

UV radiation is also a factor that decreases the viability of cells by induction of apoptosis [22]. However, UVA and
UVB radiation influences the cytosolic level of pro-apoptotic proteins such as cytochrome $c$ or caspase 3 in a various ways. As shown in this study, keratinocytes are more sensitive to radiation than fibroblasts, what is observed as a three times increase in cytochrome $c$ and caspase 3 level. This reaction may be associated with the strong ability of keratinocytes to proliferate and regenerate damage caused by UV radiation of epidermal cells [56]. Moreover, keratinocytes and fibroblasts differ from each other in the changes in the level of pro-apoptotic protein $\mathrm{Bcl}-2$ following ascorbic acid treatment. Ascorbic acid alone, as well in a mixture with rutin, significantly increases Bcl-2 level in all examined keratinocytes, but not in fibroblasts. This result is connected with the ascorbic acid function in keratinocytes to induce maturation and differentiation, and also leads to cellular aging and death [72].

The results of this study also show that UV radiation induces different responses in the level of antiapoptotic Bax protein in various types of skin cells, which is why the observed effect of ascorbic acid and rutin on the UVinduced apoptosis is slightly different. In fibroblasts, ascorbic acid and rutin decreased UV-induced expression of Bax, which was also observed in the case of ethanolinduced neurotoxicity in neuronal cells treated with rutin [76]. The opposite effect is observed in UV-irradiated keratinocytes, where ascorbic acid or rutin increased Bax expression, restoring the level similar to control cells. Furthermore, rutin has been shown to enhance arsenicinduced apoptosis via ROS-dependent p53 protein ubiquitination in human HaCaT keratinocytes [73]. However, in both cases, the combined treatment with ascorbic acid and rutin had a stronger effect to counteract the effects of UV irradiation compared with their individual use, which suggests their cooperation in apoptosis signaling.

\section{Conclusion}

In this study, we presented the cooperation of ascorbic acid and rutin in exert a cytoprotective effect on keratinocytes and fibroblasts exposed to UVA and UVB radiation. The obtained results showed that the compounds complement their action, not only in the antioxidant field, but also to support the transport and signaling functions of each other. Moreover, their combined antioxidant, antiinflammatory and antiapoptotic action makes them a potentially effective cytoprotective team against UV-induced skin damage.

Acknowledgements This study was financed by the National Science Centre Poland (NCN) Grant no. 2017/25/N/NZ7/00863. Co-author of this work, A.G. was supported by the Foundation for Polish Science (FNP). 
OpenAccess This article is distributed under the terms of the Creative Commons Attribution 4.0 International License (http://creativeco mmons.org/licenses/by/4.0/), which permits unrestricted use, distribution, and reproduction in any medium, provided you give appropriate credit to the original author(s) and the source, provide a link to the Creative Commons license, and indicate if changes were made.

\section{References}

1. Aebi H (1984) Catalase in vitro. Methods Enzymol 105:121-126

2. Afanas EVIB, Dcrozhko AI, Brodskii AV, Kostyuk VA, Potapovitch AI (1989) Chelating and free radical scavenging mechanisms of inhibitory action of rutin and quercetin in lipid peroxidation. Biochem Pharmacol 38:1763-1769

3. Al-Niaimi F, Chiang NYZ (2017) Topical vitamin C and the skin: mechanisms of action and clinical applications. J Clin Aesthet Dermatol 10:14-17

4. Al-Rejaie SS, Abuohashish HM, Alkhamees OA, Aleisa AM, Alroujayee AS (2012) Gender difference following high cholesterol diet induced renal injury and the protective role of rutin and ascorbic acid combination in Wistar albino rats. Lipids Health Dis 11:41-51

5. Annapurna A, Ansari MA, Manjunath PM (2013) Partial role of multiple pathways in infarct size limiting effect of quercetin and rutin against cerebral ischemia-reperfusion injury in rats. Eur Rev Med Pharmacol Sci 17:491-500

6. Benzie IF, Strain JJ (1996) The ferric reducing ability of plasma (FRAP) as a measure of "antioxidant power": the FRAP assay. Anal Biochem 239:70-76

7. Bernerd F, Marionnet C, Duval C (2012) Solar ultraviolet radiation induces biological alterations in human skin in vitro: relevance of a well-balanced UVA/UVB protection. Indian J Dermatol Venereol Leprol 78:15-23

8. Bouriche H, Miles EA, Selloum L, Calder PC (2005) Effect of Cleome arabica leaf extract, rutin and quercetin on soybean lipoxygenase activity and on generation of inflammatory eicosanoids by human neutrophils. Prostaglandins Leukot Essent Fatty Acids 72:195-201

9. Bradford MM (1976) A rapid and sensitive method for the quantitation of microgram quantities of protein utilizing the principle of protein-dye binding. Anal Biochem 72:248-254

10. Catani MV, Savini I, Rossi A, Melino G, Avigliano L (2005) Biological role of vitamin $\mathrm{C}$ in keratinocytes. Nutr Rev 63:81-90

11. Chojkier M, Houglum K, Solis-Herruzo J, Brenner DA (1989) Stimulation of collagen gene expression by ascorbic acid in cultured human fibroblasts. A role for lipid peroxidation? J Biol Chem 264:16957-16962

12. Choudhury AR, Sikorska E, van den Boom J, Bayer P, Popenda $€$, Szutkowski K et al (2015) Structural model of the bilitranslocase transmembrane domain supported by NMR and FRET data. PLoS One 10:e0135455

13. Coolen SA, van Buuren B, Duchateau G, Upritchard J, Verhagen $\mathrm{H}$ (2005) Kinetics of biomarkers: biological and technical validity of isoprostanes in plasma. Amino Acids 29:429-436

14. Deshmukh J, Pofahl R, Haase I (2017) Epidermal Rac1 regulates the DNA damage response and protects from UV-light-induced keratinocyte apoptosis and skin carcinogenesis. Cell Death Dis 8:e2664

15. D'Orazio J, Jarrett S, Amaro-Ortiz A, Scott T (2013) UV radiation and the skin. Int J Mol Sci 14:12222-12248

16. Eissa S, Seada LS (1998) Quantitation of bcl-2 protein in bladder cancer tissue by enzyme immunoassay: comparison with Western blot and immunohistochemistry. Clin Chem 44:1423-1429
17. Fotakis G, Timbrell JA (2006) In vitro cytotoxicity assays: comparison of LDH, neutral red, MTT and protein assay in hepatoma cell lines following exposure to cadmium chloride. Toxicol Lett 160:171-177

18. Furfaro AL, Traverso N, Domenicotti C, Piras S, Moretta L, Marinari UM et al (2016) The Nrf2/HO-1 axis in cancer cell growth and chemoresistance. Oxid Med Cell Longev 2016:1958174

19. Gabor M (1972) Pharmacologic effects of flavonoids on blood vessels. J Vasc Res 9:355-374

20. Gęgotek A, Bielawska K, Biernacki M, Dobrzyńska I, Skrzydlewska E (2017) Time-dependent effect of rutin on skin fibroblasts membrane disruption following UV radiation. Redox Biol 12:733-744

21. Gęgotek A, Bielawska K, Biernacki M, Zaręba I, Surażyński A, Skrzydlewska E (2017) Comparison of protective effect of ascorbic acid on redox and endocannabinoid systems interactions in in vitro cultured human skin fibroblasts exposed to UV radiation and hydrogen peroxide. Arch Dermatol Res 309:285-303

22. Gęgotek A, Biernacki M, Ambrożewicz E, Surażyński A, Wroński A, Skrzydlewska E (2016) The cross-talk between electrophiles, antioxidant defence and the endocannabinoid system in fibroblasts and keratinocytes after UVA and UVB irradiation. J Dermatol Sci $81: 107-117$

23. Gęgotek A, Domingues P, Skrzydlewska E (2018) Proteins involved in the antioxidant and inflammatory response in rutintreated human skin fibroblasts exposed to UVA or UVB irradiation. J Dermatol Sci 90:241-252

24. Gęgotek A, Rybałtowska-Kawałko P, Skrzydlewska E (2017) Rutin as a mediator of lipid metabolism and cellular signaling pathways interactions in fibroblasts altered by UVA and UVB radiation. Oxid Med Cell Longev 2017:4721352

25. Gęgotek A, Skrzydlewska E (2015) The role of transcription factor Nrf2 in skin cells metabolism. Arch Dermatol Res 307:385-396

26. Ghiasi M, Azadnia A, Arabieh M, Zahedi M (2012) Protective effect of rutin (vitamin p) against heme oxidation: a quantum mechanical approach. Comput Theor Chem 996:28-36

27. Griendling KK, Minieri CA, Ollerenshaw JD, Alexander RW (1994) Angiotensin II stimulates NADH and NADPH oxidase activity in cultured vascular smooth muscle cells. Circ Res 74:1141-1148

28. Guardia T, Rotelli AE, Juarez AO, Pelzer LE (2001) Antiinflammatory properties of plant flavonoids. Effects of rutin, quercetin and hesperidin on adjuvant arthritis in rat. Il Farmaco 56:683-687

29. Guo R, Wei P, Liu W (2007) Combined antioxidant effects of rutin and vitamin C in Triton X-100 micelles. J Pharm Biomed Anal 43:1580-1586

30. Han SY, Chu JX, Li GM, Zhu LS, Shi RF (2010) Effects of rutin from leaves and flowers of buckwheat (Fagopyrum esculentum Moench.) on angiotensin II-induced hypertrophy of cardiac myocytes and proliferation of fibroblasts. Lat Am J Pharm 29:137-140

31. Hazneci E, Karabulut AB, Öztürk Ç, Batçioğlu K, Doğan G, Karaca Ş et al (2005) A comparative study of superoxide dismutase, catalase, and glutathione peroxidase activities and nitrate levels in vitiligo patients. Int J Dermatol 44:636-640

32. Herrling T, Jung K, Fuchs J (2006) Measurements of UV-generated free radicals/reactive oxygen species (ROS) in skin. Spectrochim Acta A Mol Biomol Spectrosc 63:840-845

33. Hosseinzadeh H, Nassiri-Asl M (2014) Review of the protective effects of rutin on the metabolic function as an important dietary flavonoid. J Endocrinol Investig 37:783-788

34. Ivanović D, Popović A, Radulović D, Medenica M (1999) Reversed-phase ion-pair HPLC determination of some watersoluble vitamins in pharmaceuticals. J Pharm Biomed Anal 18:999-1004 
35. Je HD, Shin CY, Park SY, Yim SH, Kum C, Huh IH et al (2002) Combination of vitamin $\mathrm{C}$ and rutin on neuropathy and lung damage of diabetes mellitus rats. Arch Pharm Res 25:184-190

36. Kang JS, Kim HN, Kim JE, Mun GH, Kim YS, Cho D et al (2007) Regulation of UVB-induced IL-8 and MCP-1 production in skin keratinocytes by increasing vitamin $\mathrm{C}$ uptake via the redistribution of SVCT-1 from the cytosol to the membrane. J Investig Dermatol 127:698-706

37. Kansanen E, Jyrkkänen HK, Levonen AL (2012) Activation of stress signaling pathways by electrophilic oxidized and nitrated lipids. Free Radic Biol Med 52:973-982

38. Kim YD, Ahn KM, Yum HY, Chung HJ, Kim SM, Sung M et al (2005) Formation of basement membrane and stratification of Rabbit oral keratinocytes cultured on human acellular dermal matrix. J Korean Assoc Maxillofac Plast Reconstr Surg 27:510-522

39. Konstantinopoulos PA, Spentzos D, Fountzilas E, Francoeur N, Sanisetty S, Grammatikos AP, Hecht JL, Cannistra SA (2011) Keap1 mutations and Nrf2 pathway activation in epithelial ovarian cancer. Cancer Res 71:5081-5089

40. Kuzkaya N, Weissmann N, Harrison DG, Dikalov S (2003) Interactions of peroxynitrite, tetrahydrobiopterin, ascorbic acid, and thiols: implications for uncoupling endothelial nitricoxide synthase. J Biol Chem 278:22546-22554

41. Levine RL, Garland D, Oliver CN, Amici A, Climent I, Lenz AG et al (1990) Determination of carbonyl content in oxidatively modified proteins. Methods Enzymol 186:464-478

42. Lin JY, Selim MA, Shea CR, Grichnik JM, Omar MM, MonteiroRiviere NA et al (2003) UV photoprotection by combination topical antioxidants vitamin C and vitamin E. J Am Acad Dermatol 48:866-874

43. Lins TLBG, Cavalcante AYP, Santos JMS, Menezes VG, Barros VRP, Barberino RS et al (2017) Rutin can replace the use of three other antioxidants in the culture medium, maintaining the viability of sheep isolated secondary follicles. Theriogenology 89:263-270

44. Lisi S, Botta R, Lemmi M, Sellari-Franceschini S, Altea MA, Sisti E et al (2011) Quercetin decreases proliferation of orbital fibroblasts and their release of hyaluronic acid. J Endocrinol Investig 34:521-527

45. Lou Z, Wang H, Wang D, Zhang Y (2009) Preparation of inulin and phenols-rich dietary fibre powder from burdock root. Carbohydr Polym 78:666-671

46. Lovell MA, Xie C, Gabbita SP, Markesbery WR (2000) Decreased thioredoxin and increased thioredoxin reductase levels in Alzheimer's disease brain. Free Radic Biol Med 28:418-427

47. Łuczaj W, Gęgotek A, Skrzydlewska E (2017) Antioxidants and HNE in redox homeostasis. Free Radic Biol Med 111:87-101

48. Luo XP, Yazdanpanah M, Bhooi N, Lehotay DC (1995) Determination of aldehydes and other lipid peroxidation products in biological samples by gas chromatography-mass spectrometry. Anal Biochem 228:294-298

49. Maeso N, Garcia-Martinez D, Ruperez FJ, Cifuentes A, Barbas C (2005) Capillary electrophoresis of glutathione to monitor oxidative stress and response to antioxidant treatments in an animal model. J Chromatogr B 822:61-69

50. Marionnet C, Pierrard C, Lejeune F, Sok J, Thomas M, Bernerd $\mathrm{F}$ (2010) Different oxidative stress response in keratinocytes and fibroblasts of reconstructed skin exposed to non extreme dailyultraviolet radiation. PLoS One 5:e12059

51. Milde J, Elstner EF, Grassmann J (2004) Synergistic inhibition of low-density lipoprotein oxidation by rutin, $\gamma$-terpinene, and ascorbic acid. Phytomedicine 11:105-113

52. Misra HP, Fridovich I (1972) The role of superoxide anion in the autoxidation of epinephrine and a simple assay for superoxide dismutase. J Biol Chem 247:3170-3175
53. Mize CE, Longdon RG (1962) Hepatic glutathione reductase. Purification and general kinetic properties. J Biol Chem 237:1589-1595

54. Muthusamy V, Piva TJ (2010) The UV response of the skin: a review of the MAPK, NFKB and TNF $\alpha$ signal transduction pathways. Arch Dermatol Res 302:5-17

55. Nègre-Salvayre A, Mabile L, Delchambre J, Salvayre R (1995) $\alpha$-Tocopherol, ascorbic acid, and rutin inhibit synergistically the copper-promoted LDL oxidation and the cytotoxicity of oxidized LDL to cultured endothelial cells. Biol Trace Elem Res 47:81-91

56. Ojeh N, Akgül B, Tomic-Canic M, Philpott M, Navsaria H (2017) In vitro skin models to study epithelial regeneration from the hair follicle. PLoS One 12:e0174389

57. Orabi SA, Abdelhamid MT (2016) Protective role of $\alpha$-tocopherol on two Vicia faba cultivars against seawater-induced lipid peroxidation by enhancing capacity of anti-oxidative system. J Saudi Soc Agric Sci 15:145-154

58. Paglia DE, Valentine WN (1967) Studies on the quantitative and qualitative characterization of erythrocyte glutathione peroxidase. J Lab Clin Med 70:158-169

59. Passamonti S, Vrhovsek U, Mattivi F (2002) The interaction of anthocyanins with bilitranslocase. Biochem Biophys Res Commun 296:631-636

60. Peng F, Xu P, Zhao BY, Zong MH, Lou WY (2018) The application of deep eutectic solvent on the extraction and in vitro antioxidant activity of rutin from Sophora japonica bud. J Food Sci Technol 55:2326-2333

61. Potapovich AI, Lulli D, Fidanza P, Kostyuk VA, De Luca C, Pastore $S$ et al (2011) Plant polyphenols differentially modulate inflammatory responses of human keratinocytes by interfering with activation of transcription factors NFKB and AhR and EGFR-ERK pathway. Toxicol Appl Pharmacol 255:138-149

62. Prajda N, Weber G (1975) Malignant transformation-linked imbalance: decreased xanthine oxidase activity in hepatomas. FEBS Lett 59:245-259

63. Pullar JM, Carr AC, Vissers M (2017) The roles of vitamin C in skin health. Nutrients 9:866-893

64. Rani V, Deep G, Singh RK, Palle K, Yadav UC (2016) Oxidative stress and metabolic disorders: pathogenesis and therapeutic strategies. Life Sci 148:183-193

65. Re R, Pellegrini N, Proteggente A, Pannala A, Yang M, RiceEvans C (1999) Antioxidant activity applying an improved ABTS radical cation decolorization assay. Free Radic Biol Med 26:1231-1237

66. Rekha C, Poornima G, Manasa M, Abhipsa V, Devi JP, Kumar VHT et al (2012) Ascorbic acid, total phenol content and antioxidant activity of fresh juices of four ripe and unripe citrus fruits. Chem Sci Trans 1:303-310

67. Ricciotti E, FitzGerald GA (2011) Prostaglandins and inflammation. Arterioscler Thromb Vasc Biol 31:986-1000

68. Roduit R, Schorderet DF (2008) MAP kinase pathways in UVinduced apoptosis of retinal pigment epithelium ARPE19 cells. Apoptosis 13:343-353

69. Sagun KC, Cárcamo JM, Golde DW (2005) Vitamin C enters mitochondria via facilitative glucose transporter 1 (Glut1) and confers mitochondrial protection against oxidative injury. FASEB J 19:1657-1667

70. Salgado RM, Cruz-Castañeda O, Elizondo-Vázquez F, Pat L, De la GA, Cano-Colín S., et al (2017) Maltodextrin/ascorbic acid stimulates wound closure by increasing collagen turnover and TGF- $\beta 1$ expression in vitro and changing the stage of inflammation from chronic to acute in vivo. J Tissue Viability 26:131-137

71. Savini I, Rossi A, Duranti G, Avigliano L, Catani MV, Melino G (2002) Characterization of keratinocyte differentiation induced by ascorbic acid: protein kinase $\mathrm{C}$ involvement and vitamin $\mathrm{C}$ homeostasis1. J Invest Dermatol 118:372-379 
72. Seo A, Kitagawa N, Matsuura T, Sato H, Inai T (2016) Formation of keratinocyte multilayers on filters under airlifted or submerged culture conditions in medium containing calcium, ascorbic acid, and keratinocyte growth factor. Histochem Cell Biol 146:585-597

73. Shen SC, Lee WR, Yang LY, Tsai HH, Yang LL, Chen YC (2012) Quercetin enhancement of arsenic-induced apoptosis via stimulating ROS-dependent $\mathrm{p} 53$ protein ubiquitination in human $\mathrm{HaCaT}$ keratinocytes. Exp Dermatol 21:370-375

74. Si YX, Yin SJ, Oh S, Wang ZJ, Ye S, Yan L et al (2012) An integrated study of tyrosinase inhibition by rutin: progress using a computational simulation. J Biomol Struct Dyn 29:999-1012

75. Skehan P, Storeng R, Scudiero D, Monks A, McMahon J, Vistica $D$ et al (1990) New colorimetric cytotoxicity assay for anticancerdrug screening. JNCI J Natl Cancer Inst 82:1107-1112

76. Song K, Kim S, Na JY, Park JH, Kim JK, Kim JH, Kwon J (2014) Rutin attenuates ethanol-induced neurotoxicity in hippocampal neuronal cells by increasing aldehyde dehydrogenase 2 . Food Chem Toxicol 72:228-233

77. Sthijns MM, Schiffers PM, Janssen GM, Lemmens KJ, Ides B, Vangrieken $\mathrm{P}$ et al (2017) Rutin protects against $\mathrm{H}_{2} \mathrm{O}_{2}$-triggered impaired relaxation of placental arterioles and induces Nrf2-mediated adaptation in human umbilical vein endothelial cells exposed to oxidative stress. Biochim Biophys Acta 1861:1177-1189

78. Stipcevic T, Piljac J, Berghe DV (2006) Effect of different flavonoids on collagen synthesis in human fibroblasts. Plant Foods Hum Nutr 61:27-32

79. Sunada S, Fujisawa H, Cartwright IM, Maeda J, Brents CA, Mizuno K et al (2014) Monoglucosyl rutin as a potential radioprotector in mammalian cells. Mol Med Rep 10:10-14

80. Sykes JA, McCormac FX, O'Breien TJ (1978) Preliminary study of the superoxide dismutase content of some human tumors. Cancer Res 38:2759-2762
81. Tanimoto A, Ueda Y, Kimoto Y, Amano W, Kabashima K (2016) U.S. Patent Application No. 15/072013

82. Tarumoto T, Nagai T, Ohmine K, Miyoshi T, Nakamura M, Kondo $\mathrm{T}$ et al (2004) Ascorbic acid restores sensitivity to imatinib via suppression of Nrf2-dependent gene expression in the imatinibresistant cell line. Exp Hematol 32:375-381

83. Tebbe B, Wu S, Geilen CC, Eberle J, Kodelja V, Orfanos CE (1997) L-ascorbic acid inhibits UVA-induced lipid peroxidation and secretion of IL- $1 \alpha$ and IL-6 in cultured human keratinocytes in vitro. J Investig Dermatol 108:302-306

84. Tran NQ, Joung YK, Lih E, Park KD (2011) In situ forming and rutin-releasing chitosan hydrogels as injectable dressings for dermal wound healing. Biomacromolecules 12:2872-2880

85. Vatassery GT, Brin MF, Fahn S, Kayden HJ, Traber MG (1988) Effect of high doses of dietary vitamin $\mathrm{E}$ on the concentrations of vitamin $\mathrm{E}$ in several brain regions, plasma, liver, and adipose tissue of rats. J Neurochem 51:621-623

86. Wagner AE, Huebbe P, Konishi T, Rahman MM, Nakahara M, Matsugo S, Rimbach G (2008) Free radical scavenging and antioxidant activity of ascorbigen versus ascorbic acid: studies in vitro and in cultured human keratinocytes. J Agric Food Chem 56:11694-11699

87. Zhu AS, Li A, Ratliff TS, Melsom M, Garza LA (2017) After skin wounding, noncoding dsRNA coordinates prostaglandins and Wnts to promote regeneration. J Investig Dermatol 137:1562-1568

Publisher's Note Springer Nature remains neutral with regard to jurisdictional claims in published maps and institutional affiliations. 